\title{
QUASI-SYMMETRIES OF DETERMINANTAL POINT PROCESSES ${ }^{1}$
}

\author{
By AlEXANDER I. Bufetov \\ Aix-Marseille Université, CNRS, Centrale Marseille, Institut de Mathématiques \\ de Marseille, UMR 7373, Steklov Mathematical Institute of RAS, Institute for \\ Information Transmission Problems, National Research University Higher School \\ of Economics and Saint-Petersburg State University
}

\begin{abstract}
The main result of this paper is that determinantal point processes on $\mathbb{R}$ corresponding to projection operators with integrable kernels are quasiinvariant, in the continuous case, under the group of diffeomorphisms with compact support (Theorem 1.4); in the discrete case, under the group of all finite permutations of the phase space (Theorem 1.6). The Radon-Nikodym derivative is computed explicitly and is given by a regularized multiplicative functional. Theorem 1.4 applies, in particular, to the sine-process, as well as to determinantal point processes with the Bessel and the Airy kernels; Theorem 1.6 to the discrete sine-process and the Gamma kernel process. The paper answers a question of Grigori Olshanski.
\end{abstract}

\section{Introduction.}

1.1. Outline of the main results. Olshanski [22] established the quasiinvariance of the determinantal measure corresponding to the Gamma kernel under the group of finite permutations of $\mathbb{Z}$ and expressed the Radon-Nikodym derivative as a multiplicative functional. Ghosh and Peres [11,13] showed, for the Ginibre ensemble and the Gaussian zero process, that the conditional distribution of particles in a bounded domain, with the configuration fixed in the exterior, is equivalent to the Lebesgue measure.

In this paper, we take a determinantal point process on $\mathbb{R}$ governed by an orthogonal projection onto a closed subspace $L$ with the following property: given

Received October 2015; revised May 2017.

${ }^{1}$ Supported by the A*MIDEX project (no. ANR-11-IDEX-0001-02) funded by the programme "Investissements d'Avenir" of the Government of the French Republic, managed by the French National Research Agency (ANR). This project has received funding from the European Research Council (ERC) under the European Union's Horizon 2020 research and innovation programme (grant agreement No 647133 (ICHAOS)) and has also been funded by Grant MD 5991.2016.1 of the President of the Russian Federation, by the Russian Academic Excellence Project "5-100" and by the Chaire Gabriel Lamé at the Chebyshev Laboratory.

MSC2010 subject classifications. Primary 60G55; secondary 22E66, 60B10, 60B15, 33C10.

Key words and phrases. Determinantal point processes, Gibbs property, Palm measures, integrable kernels, multiplicative functionals. 
$p \in \mathbb{R}$ and $\varphi \in L$ satisfying $\varphi(p)=0$, we have

$$
\frac{\varphi(x)}{x-p} \in L
$$

see Assumption 2 below for the precise formulation.

In particular, as we check below, Assumption 2 holds for projections governed by kernels admitting an integrable representation

$$
\frac{A(x) B(y)-B(x) A(y)}{x-y} .
$$

The term integrable comes from the connection with the theory of integrable systems discovered by Its, Izergin, Korepin and Slavnov in [15].

The main results of this paper, Theorems 1.4 and 1.6, establish that, under some additional assumptions, the measure class of the corresponding determinantal measures is preserved, in the continuous case, under the group of diffeomorphisms with compact support (Theorem 1.4); in the discrete case, under the group of finite permutations of the phase space (Theorem 1.6). The key step in the proof is the equivalence of reduced Palm measures corresponding to different $l$-tuples of points $\left(p_{1}, \ldots, p_{l}\right),\left(q_{1}, \ldots, q_{l}\right)$ in the phase space; the corresponding Radon-Nikodym derivative is the regularized multiplicative functional corresponding to the function

$$
\left(\frac{\left(x-p_{1}\right) \cdots\left(x-p_{l}\right)}{\left(x-q_{1}\right) \cdots\left(x-q_{l}\right)}\right)^{2} .
$$

The Radon-Nikodym derivative thus has similar form for all the processes with integrable kernels; the normalizing constants do, of course, depend on the specific process.

Olshanski [22] proves the quasi-invariance of the Gamma-kernel process by a limit transition from finite-dimensional approximations. The argument in this paper is direct: first, it is shown that the Palm subspaces corresponding to conditioning at points $p$ and $q$ are taken one to the other by multiplication by the function $(x-p) /(x-q)$; after which, the proof is completed using a general result of $[5,7]$ that multiplying the range of the projection operator $\Pi$ inducing a determinantal measure $\mathbb{P}_{\Pi}$ by a function $g$, corresponds, under certain additional assumptions, to taking the product of the determinantal measure $\mathbb{P}_{\Pi}$ by the multiplicative functional $\Psi_{g}$ induced by the function $g$. The key technical step is the regularization of divergent multiplicative functionals.

This paper is devoted to determinantal point processes governed by orthogonal projections; in the case of contractions, quasi-invariance is due to Camilier and Decreusefond [9]; note that in their case the Radon-Nikodym derivative exhibits a much more sensitive dependence on the specific kernel.

\subsection{Projection operators and determinantal point processes.}

1.2.1. Operators and kernels. Let $\mu$ be a $\sigma$-finite Borel measure on $\mathbb{R}$; for example, $\mu$ can be the Lebesgue measure on $\mathbb{R}$ or on $\mathbb{R}_{+}$or else the counting mea- 
sure on $\mathbb{Z}$. The inner product in $L_{2}(\mathbb{R}, \mu)$ will be denoted $\langle$,$\rangle . Let L \subset L_{2}(\mathbb{R}, \mu)$ be a closed subspace, and let $\Pi$ be the corresponding operator of orthogonal projection. We assume that the operator $\Pi$ is locally of trace class and admits a kernel, for which, slightly abusing notation, we keep the same symbol $\Pi$. We let $\mathbb{P}_{\Pi}$ be the determinantal measure on the space $\operatorname{Conf}(\mathbb{R})$ of configurations on $\mathbb{R}$ induced by the operator $\Pi$ (see Section 2 below for detailed definitions). All kernels considered in this paper will always be supposed to satisfy the following

ASSUMPTION 1. There exists a set $U \subset \mathbb{R}$, satisfying $\mu(\mathbb{R} \backslash U)=0$, such that

1. For any $q \in U$, the function $v_{q}(x)=\Pi(x, q)$ lies in $L_{2}(\mathbb{R}, \mu)$ and for any $f \in L_{2}(\mathbb{R}, \mu)$ we have

$$
\Pi f(q)=\left\langle f, v_{q}\right\rangle .
$$

In particular, all functions in $L$ are defined everywhere on $U$.

2. The diagonal values $\Pi(q, q)$ of the kernel $\Pi$ are defined for all $q \in U$. We have $\left\langle v_{q}, v_{q}\right\rangle=\Pi(q, q)$, and, for any bounded Borel subset $B \subset \mathbb{R}$, we have

$$
\operatorname{tr}\left(\chi_{B} \Pi \chi_{B}\right)=\int_{B} \Pi(q, q) d \mu(q) .
$$

3. For any $q \in U$ and any $\varphi \in L$ satisfying $\varphi(q)=0$, we have

$$
\frac{\varphi(x)}{x-q} \in L_{2}(\mathbb{R}, \mu) \text {. }
$$

The first assumptions automatically hold, for instance, for continuous reproducing kernels; the subset $U$ is introduced in order to allow the consideration of kernels defined on subsets of $\mathbb{R}$ such as, for example, the Bessel kernel defined on $\mathbb{R}_{+}$(see Tracy-Widom [32]).

The last condition is automatically satisfied once the kernel is sufficiently smooth: indeed, let $\varphi \in L$ have norm 1 and be such that $\varphi(q)=0$, let

$$
\Pi^{q}(x, y)=\Pi(x, y)-\frac{\Pi(x, q) \Pi(q, y)}{\Pi(q, q)}
$$

be the kernel of the orthogonal projection onto the space $L(q)$, the orthogonal complement of $v_{q}$ in $L$. Finally, let $\tilde{\Pi}$ be the kernel of the orthogonal projection onto the orthogonal complement of $\varphi$ in $L(q)$. For any $x \in U$, by definition, we have

$$
\Pi^{q} v_{x}=\left\langle v_{x}, \varphi\right\rangle \varphi+\tilde{\Pi} v_{x},
$$

whence, taking the inner product with $v_{x}$, we obtain

$$
\Pi^{q}(x, x)=|\varphi(x)|^{2}+\left\langle\tilde{\Pi} v_{x}, v_{x}\right\rangle .
$$


Using (3) and the smoothness of the kernel $\Pi$, we now write the Taylor series for $\Pi^{q}(x, x)$ in a small neighbourhood of $q$ and obtain $\Pi^{q}(x, x)=O\left(|x-q|^{2}\right)$, whence also $|\varphi(x)|=O(|x-q|)$, which implies the desired last condition of Assumption 1.

1.2.2. Palm subspaces. Given $l$ distinct points $q_{1}, \ldots, q_{l} \in \mathbb{R}$, we set

$$
L\left(q_{1}, \ldots, q_{l}\right)=\left\{\varphi \in L: \varphi\left(q_{1}\right)=\cdots=\varphi\left(q_{l}\right)=0\right\} ;
$$

we denote $\Pi^{q_{1}, \ldots, q_{l}}$ the operator of orthogonal projection onto the subspace $L\left(q_{1}, \ldots, q_{l}\right)$, and we denote by $\mathbb{P}_{\Pi}^{q_{1}, \ldots, q_{l}}$ the determinantal measure governed by the projection $\Pi^{q_{1}, \ldots, q_{l}}$; by the Shirai-Takahashi theorem, $\mathbb{P}_{\Pi}^{q_{1}, \ldots, q_{l}}$ is the reduced Palm measure of the determinantal measure $\mathbb{P}_{\Pi}$ with respect to the points $q_{1}, \ldots, q_{l}$ (the definition and properties of Palm measures are recalled in detail in Section 2 below).

1.2.3. The main assumption. We now formulate our main assumption on our projection operators.

AsSUMPTION 2. If $p \in U$ and $\varphi \in L$ are such that $\varphi(p)=0$, then there exists $\psi \in L$ such that

$$
\varphi=(x-p) \psi
$$

REMARK. If the measure $\mu$ does not admit atoms, we can simply say that for $p \in U$ and $\varphi \in L$ satisfying $\varphi(p)=0$ we have (1). In the discrete case, if $\psi$ satisfies (4), then for any $\alpha \in \mathbb{R}$, letting $\delta_{p}$ be the delta-function at $p$, we see that the function $\psi+\alpha \delta_{p}$ also satisfies (4). Our requirement is that one of these functions does belong to $L$. Induction gives the following proposition.

Proposition 1.1. For any $l \in \mathbb{N}$, if $p_{1}, \ldots, p_{l} \in U$ are distinct and $\varphi \in L$ satisfies $\varphi\left(p_{1}\right)=\cdots=\varphi\left(p_{l}\right)=0$, then there exists $\psi \in L$ such that

$$
\varphi=\prod_{i=1}^{l}\left(x-p_{i}\right) \psi .
$$

1.2.4. Change of variables. Given a Borel measure $\mu$ on a Borel space $\mathscr{X}$ and a Borel automorphism $T$ of $\mathscr{X}$, denote by $\mu \circ T$ the measure defined by $\mu \circ T(\mathscr{Z})=\mu(T(\mathscr{Z}))$ for all Borel subsets $\mathscr{Z} \subset \mathscr{X}$. Since $T$ is invertible, the measure $\mu \circ T$ is well defined and, for any $\mu$-integrable Borel function $f$ on $\mathscr{X}$, satisfies

$$
\int_{\mathscr{X}} f \circ T d \mu \circ T=\int_{\mathscr{X}} f d \mu .
$$

For a nonnegative Borel function $f$, we also have $(f \mu) \circ T=(f \circ T)(\mu \circ T)$. 
Let $F$ be a Borel automorphism of $\mathbb{R}$ sending bounded sets to bounded sets. The automorphism $F$ acts on $\operatorname{Conf}(\mathbb{R})$ by sending a configuration $X$ to the configuration $F(X)=\{F(x), x \in X\}$; slightly abusing notation, we keep the same symbol $F$ for this induced action.

Assume additionally that the measure $\mu$ is quasi-invariant under $F$. Then the measure $\mathbb{P}_{\Pi} \circ F^{-1}$ is determinantal with kernel

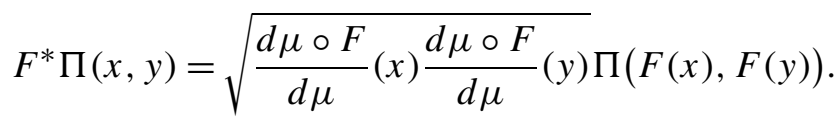

The kernel $F^{*} \Pi$ induces the operator of orthogonal projection onto the subspace

$$
L_{F^{*} \Pi}=F_{*} L=\left\{\sqrt{F^{\prime}} \cdot \varphi \circ F, \varphi \in L\right\} .
$$

1.3. Integrable kernels. A key particular case is when our kernel $\Pi$ has integrable form: there exists an open set $U \subset \mathbb{R}$ satisfying $\mu(\mathbb{R} \backslash U)=0$ and linearly independent smooth functions $A, B$ defined on $U$ such that

$$
\Pi(x, y)=\frac{A(x) B(y)-A(y) B(x)}{x-y}, \quad x \neq y .
$$

We assume that the functions $A, B$ never simultaneously take value 0 on $U$. For $p \in U$ we have

$$
v_{p}(x)=\frac{A(p) B(x)-B(p) A(x)}{p-x} .
$$

We have $v_{p} \in L_{2}(\mathbb{R}, \mu)$ for any $p \in U$ and for any $\varphi \in L_{2}(\mathbb{R}, \mu)$ we have

$$
\Pi \varphi(p)=\left\langle\varphi, v_{p}\right\rangle \text {. }
$$

We consider two cases:

1. the continuous case: for any $p \in \mathbb{R}, \mu(\{p\})=0$;

2. the discrete case: $\mu$ is the counting measure on a countable subset $E \subset \mathbb{R}$ without accumulation points.

In the continuous case, we make the additional requirement

$$
\Pi(x, x)=A^{\prime}(x) B(x)-A(x) B^{\prime}(x),
$$

on diagonal values of the kernel $\Pi$; in the discrete case, when the measure $\mu$ is the counting measure on a countable subset $E \subset \mathbb{R}$ without accumulation points, the integrability assumption only concerns off-diagonal entries of the kernel $\Pi(x, y)$, and the smoothness assumption is not needed: $A, B$ are just arbitrary functions defined on $E$. Note also that the third requirement of Assumption 1 is only needed in the continuous case.

Note also that the functions $A, B$ in the definition of integrability are not unique: for example, if one makes a linear unimodular change of variable

$$
(A, B) \rightarrow\left(\alpha_{11} A+\alpha_{12} B, \alpha_{21} A+\alpha_{22} B\right), \quad \alpha_{11} \alpha_{22}-\alpha_{12} \alpha_{21}=1,
$$

then the formula (6) remains valid. 
The following proposition plays a crucial rôle in what follows.

PROPOSITION 1.2. An operator of orthogonal projection admitting an integrable kernel satisfies Assumption 2.

1.4. The main result in the continuous case. In this subsection, we assume that the measure $\mu$ does not admit atoms. Let $p_{1}, \ldots, p_{l}, q_{1}, \ldots, q_{l} \in \mathbb{R}$ be distinct. For $R>0, \varepsilon>0$ and a configuration $X$ on $\mathbb{R}$ write

$$
\begin{aligned}
& \bar{\Psi}_{R, \varepsilon}\left(p_{1}, \ldots, p_{l} ; q_{1}, \ldots, q_{l} ; X\right) \\
& =C(R, \varepsilon) \times \prod_{x \in X,|x| \leq R, \min \left|x-q_{i}\right| \geq \varepsilon} \prod_{i=1}^{l}\left(\frac{x-p_{i}}{x-q_{i}}\right)^{2},
\end{aligned}
$$

where the constant $C(R, \varepsilon)$ is chosen in such a way that

$$
\int_{\operatorname{Conf}(\mathbb{R})} \bar{\Psi}_{R, \varepsilon}\left(p_{1}, \ldots, p_{l} ; q_{1}, \ldots, q_{l} ; X\right) d \mathbb{P}_{\Pi}^{q_{1}, \ldots, q_{l}}=1 .
$$

We will need the following.

Assumption 3. The kernel $\Pi$ satisfies

$$
\int_{\mathbb{R}} \frac{\Pi(x, x)}{1+x^{2}} d \mu(x)<+\infty
$$

PROPOSITION 1.3. If the kernel $\Pi$ of an orthogonal projection operator satisfies Assumptions 1, 2, 3, then the limit

(11) $\bar{\Psi}\left(p_{1}, \ldots, p_{l} ; q_{1}, \ldots, q_{l} ; X\right)=\lim _{R \rightarrow \infty, \varepsilon \rightarrow 0} \bar{\Psi}_{R, \varepsilon}\left(p_{1}, \ldots, p_{l} ; q_{1}, \ldots, q_{l} ; X\right)$

exists in $L_{1}\left(\operatorname{Conf}(\mathbb{R}), \mathbb{P}_{\Pi^{q_{1}}, \ldots, q_{l}}\right)$.

REMARK. The limit in $R$ and $\varepsilon$ in (11) is understood in the totality of variables, in particular, regardless of order.

THEOREM 1.4. Let $\mu$ be a continuous measure on $\mathbb{R}$. Let $\Pi$ be a kernel inducing a locally trace-class operator of orthogonal projection in $L_{2}(\mathbb{R}, \mu)$ and satisfying Assumptions 1, 2, 3. Then

1. For any $l \in \mathbb{N}$ and two l-tuples of distinct points

$$
p_{1}, \ldots, p_{l}, q_{1}, \ldots, q_{l} \in U
$$

we have

$$
\frac{d \mathbb{P}_{\Pi}^{p_{1}, \ldots, p_{l}}}{d \mathbb{P}_{\Pi}^{q_{1}, \ldots, q_{l}}}=\bar{\Psi}\left(p_{1}, \ldots, p_{l} ; q_{1}, \ldots, q_{l}\right) .
$$

2. Let $F: \mathbb{R} \rightarrow \mathbb{R}$ be a Borel automorphism acting as the identity beyond a bounded open set $V \subset \mathbb{R}$ and such that $\mu$ is quasi-invariant under $F$. For $\mathbb{P}_{\Pi^{-}}$ 
almost every configuration $X \in \operatorname{Conf}(\mathbb{R}), X \cap V=\left\{q_{1}, \ldots, q_{l}\right\}$, we have

$$
\begin{aligned}
\frac{d \mathbb{P}_{\Pi \circ F}}{d \mathbb{P}_{\Pi}}(X)= & \bar{\Psi}\left(F\left(q_{1}\right), \ldots, F\left(q_{l}\right) ; q_{1}, \ldots, q_{l} ; X\right) \\
& \times \frac{\operatorname{det}\left(\Pi\left(F\left(q_{i}\right), F\left(q_{j}\right)\right)\right)_{i, j=1, \ldots, l}}{\operatorname{det}\left(\Pi\left(q_{i}, q_{j}\right)\right)_{i, j=1, \ldots, l}} \\
& \times \frac{d \mu \circ F}{d \mu}\left(q_{1}\right) \ldots \frac{d \mu \circ F}{d \mu}\left(q_{l}\right) .
\end{aligned}
$$

REMARK. The open set $V$ can be chosen in many ways; the resulting value of the Radon-Nikodym derivative is of course the same.

For example, Theorem 1.4 applies to the sine-process as well as to the Airy and Bessel point processes of Tracy and Widom [31, 32]. The results of this paper were announced without proof in [8].

REMARK. In the sequel to this paper, in joint work with Yanqi Qiu we obtain quasi-invariance results for determinantal point processes corresponding to Hilbert spaces of holomorphic functions on the plane and on the disc.

1.5. The main result in the discrete case. The main result is similar in the discrete case except that we also need to consider measures conditional on the absence of particles and that, in order to ensure quasi-invariance of our measures under the infinite symmetric group, we impose the extra restriction that our subspace $L$ not contain functions with finite support.

Let $E \subset \mathbb{R}$ be a countable subset without accumulation points, endowed with the counting measure. The analogue of Assumption 3 in the discrete case is the following assumption.

Assumption 4. The subset $E$ satisfies

$$
\sum_{n \in E} \frac{1}{1+n^{2}}<+\infty
$$

Let $\Pi$ be a kernel inducing an operator of orthogonal projection onto a subspace $L \subset L_{2}(E)$, and let $\mathbb{P}_{\Pi}$ be the corresponding determinantal measure on the space $\operatorname{Conf}(E)$ of configurations on $E$. The infinite symmetric group naturally acts on $E$ by finite permutations and induces the corresponding natural action on $\operatorname{Conf}(E)$. Given $l \in \mathbb{N}, m<l$ and an $l$-tuple $\left(p_{1}, \ldots, p_{l}\right)$ of distinct points in $E$ such that there does not exist a nonzero function in $L$ supported on the set $\left\{p_{1}, \ldots, p_{l}\right\}$, we introduce a closed subspace $L\left(p_{1}, \ldots, p_{m}, \breve{p}_{m+1}, \ldots, \breve{p}_{l}\right)$ by the formula

$$
\begin{aligned}
& L\left(p_{1}, \ldots, p_{m}, \breve{p}_{m+1}, \ldots, \breve{p}_{l}\right) \\
& \quad=\left\{\chi_{E \backslash\left\{p_{m+1}, \ldots, p_{l}\right\}} \varphi: \varphi \in L, \varphi\left(p_{1}\right)=\cdots=\varphi\left(p_{m}\right)=0\right\} .
\end{aligned}
$$


Let $\Pi^{p_{1}, \ldots, p_{m}, \breve{p}_{m+1}, \ldots, \breve{p}_{l}}$ be the corresponding orthogonal projection operator, $\mathbb{P}_{\Pi^{p_{1}}, \ldots, p_{m}, \breve{p}_{m+1}, \ldots, \breve{p}_{l}}$ the corresponding determinantal measure. The determinantal

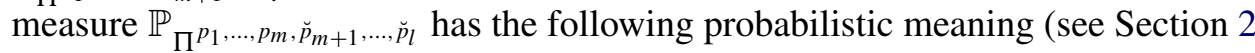
below for details): consider the conditional measure of $\mathbb{P}_{\Pi}$ with respect to the condition that there be particles at positions $p_{1}, \ldots, p_{m}$ and holes in positions $p_{m+1}, \ldots, p_{l}$; now remove the particles at $p_{1}, \ldots, p_{m}$; the resulting "reduced"

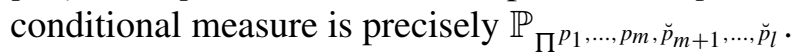

Take $R>0, m \leq l$, a permutation $\sigma$ of the points $p_{1}, \ldots, p_{l}$, and define

$$
\bar{\Psi}_{R}\left(p_{1}, \ldots, p_{l}, m, \sigma ; X\right)=C_{R} \prod_{x \in X:|x| \leq R} \prod_{i=1}^{m}\left(\frac{x-\sigma\left(p_{i}\right)}{x-p_{i}}\right)^{2} \chi_{E \backslash\left\{p_{1}, \ldots, p_{l}\right\}}(x),
$$

where the positive constant $C_{R}$ is chosen in such a way that

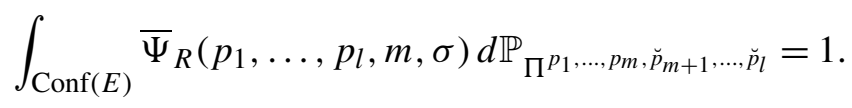

REMARK. Since the subspace $L$ does not admit functions supported on $\left\{p_{1}, \ldots, p_{l}\right\}$, the set of configurations having no particles in positions $p_{1}, \ldots, p_{l}$ has positive probability, consequently, our functional $\bar{\Psi}_{R}\left(p_{1}, \ldots, p_{l}, m, \sigma ; X\right)$ is positive with positive probability.

PROPOSITION 1.5. Let $E$ be a countable subset of $\mathbb{R}$ without accumulation points satisfying Assumption 4 . Let $\Pi$ be a kernel inducing an operator of orthogonal projection on $L_{2}(E)$. Let $p_{1}, \ldots, p_{l} \in E$ be distinct points such that there does not exist a nonzero function in $L$ supported on the set $\left\{p_{1}, \ldots, p_{l}\right\}$. Then, for any $m \leq l$ and any permutation $\sigma$ of $p_{1}, \ldots, p_{l}$, the limit

$$
\bar{\Psi}\left(p_{1}, \ldots, p_{l}, m, \sigma\right)=\lim _{R \rightarrow \infty} \bar{\Psi}_{R}\left(p_{1}, \ldots, p_{l}, m, \sigma\right)
$$

exists in $L_{1}\left(\operatorname{Conf}(E), \mathbb{P}_{\Pi^{p_{1}, \ldots, p m}, \breve{p}_{m+1}, \ldots, \breve{p}_{l}}\right)$.

Let $\mathbf{C}\left(p_{1}, \ldots, p_{m}, \breve{p}_{m+1}, \ldots, \breve{p}_{l}\right)$ be the set of configurations on $E$ containing exactly one particle in each of the positions $p_{1}, \ldots, p_{m}$ and no particles in the positions $p_{m+1}, \ldots, p_{l}$.

We are now ready to formulate the main result in the discrete case, the quasiinvariance of determinantal measures with integrable kernels under the natural action of the infinite symmetric group on $\operatorname{Conf}(E)$. Given a permutation $\sigma$ of points $p_{1}, \ldots, p_{l}$ of the set $E$, slightly abusing notation, we use the same symbol both for the bijection of $E$ that acts as $\sigma$ on $\left\{p_{1}, \ldots, p_{l}\right\}$ and as the identity on $E \backslash\left\{p_{1}, \ldots, p_{l}\right\}$ and the automorphism induced by this bijection on the space $\operatorname{Conf}(E)$ of configurations on $E$.

THEOREM 1.6. Let $E$ be a countable subset of $\mathbb{R}$ without accumulation points satisfying (4). Let $\Pi$ be a kernel inducing an operator of orthogonal projection 
onto a closed subspace $L \subset L_{2}(E)$ and satisfying Assumptions 1,2 . Let $p_{1}, \ldots, p_{l}$ be distinct points in $E$ such that there does not exist a nonzero function in $L$ supported on the set $\left\{p_{1}, \ldots, p_{l}\right\}$. Then for any $m \leq l$, any permutation $\sigma$ of the points

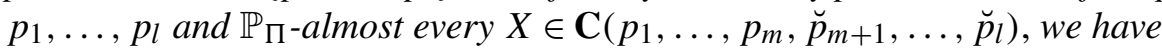

$$
\frac{d \mathbb{P}_{\Pi} \circ \sigma}{d \mathbb{P}_{\Pi}}(X)=\bar{\Psi}\left(p_{1}, \ldots, p_{l}, m, \sigma ; X\right) \times \frac{\operatorname{det}\left(\Pi\left(\sigma\left(p_{i}\right), \sigma\left(p_{j}\right)\right)\right)_{i, j=1, \ldots, m}}{\operatorname{det}\left(\Pi\left(p_{i}, p_{j}\right)\right)_{i, j=1, \ldots, m}}
$$

In particular, if the subspace L does not contain functions supported on finite sets, then the measure $\mathbb{P}_{\Pi}$ is quasi-invariant under the natural action of the infinite symmetric group on $\operatorname{Conf}(E)$.

For example, the discrete sine-process of Borodin, Okounkov and Olshanski [2] as well as the Gamma kernel process of Borodin and Olshanski [3] satisfy all the assumptions of Theorem 1.6.

REMARK. By the theorem of Ghosh [12], the sine-process, discrete or continuous, is rigid: if, for a bounded subset $B$ and a configuration $X$, we let $\#_{B}(X)$ stand for the number of particles of $X$ lying in $B$, and, for any Borel subset $C$ we let $\mathcal{F}_{C}$ be the $\sigma$-algebra generated by all random variables of the form $\#_{B}, B \subset C$, then, for any bounded $B$, the random variable $\#_{B}$ is measurable with respect to the completion, under the sine-process, of the sigma-algebra $\mathcal{F}_{B^{c}}$, where $B^{c}$ stands for the complement of $B$. As Lyons, developing the method of [1], showed in Theorem 7.15 of [19], the tail sigma-algebra of the discrete sine-process is trivial. It follows now that the symmetric sigma-algebra of the sine-process is trivial as well: in other words, the discrete sine-process is ergodic with respect to the action of the infinite symmetric group. This argument holds, of course, for any rigid point process.

To further illustrate Theorem 1.6, we now write the Radon-Nikodym derivative for a transposition of two points $p, q \in E$. Set

$$
L(p, \breve{q})=\left\{\chi_{E \backslash\{p, q\}} \varphi, \varphi \in L, \varphi(p)=0\right\},
$$

and let $\mathbb{P}_{\Pi}^{p, \breve{q}}$ be the determinantal measure corresponding to the operator of orthogonal projection onto the subspace $L(p, \breve{q})$. The subspace $L(q, \breve{p})$ and the measure $\mathbb{P}_{\Pi}^{q, \breve{p}}$ are defined in the same way. Write

$$
\bar{\Psi}_{N}(p, q ; X)=C_{p, q} \times \prod_{x \in X,|x| \leq N}\left(\frac{x-p}{x-q}\right)^{2},
$$

where the constant $C_{p, q}$ is chosen in such a way that

$$
\int_{\operatorname{Conf}(E)} \bar{\Psi}_{N}(p, q ; X) d \mathbb{P}_{\Pi}^{p, \breve{q}}(X)=1 .
$$

By definition, $\mathbb{P}_{\Pi}^{p, \breve{q}}$-almost all configurations $X$ on $E$ contain no particles either at $p$ or at $q$, so the function $\bar{\Psi}_{N}$ is well defined; by definition it is bounded. 
Proposition 1.7. The limit $\bar{\Psi}(p, q ; X)=\lim _{N \rightarrow \infty} \bar{\Psi}_{N}(p, q ; X)$ exists in $L_{1}\left(\operatorname{Conf}(E), \mathbb{P}_{\Pi}^{p, \breve{q}}\right)$.

The Radon-Nikodym derivative of $\mathbb{P}_{\Pi}$ under the action of the permutation $\sigma_{p q}$ is now given by the following

PROposition 1.8. For $\mathbb{P}_{\Pi}$-almost all $X \in \operatorname{Conf}(E)$ the following holds.

If $p \notin X, q \in X$, then

$$
\frac{d \mathbb{P}_{\Pi \circ \sigma_{p q}}}{d \mathbb{P}_{\Pi}}(X)=\bar{\Psi}(p, q ; X) \cdot \frac{\Pi(p, p)}{\Pi(q, q)} .
$$

If $p \in X, q \notin X$, then

$$
\frac{d \mathbb{P}_{\Pi} \circ \sigma_{p q}}{d \mathbb{P}_{\Pi}}(X)=\bar{\Psi}(q, p ; X) \cdot \frac{\Pi(q, q)}{\Pi(p, p)} .
$$

If $p, q \in X$ or $p, q \notin X$, then

$$
\frac{d \mathbb{P}_{\Pi} \circ \sigma_{p q}}{d \mathbb{P}_{\Pi}}(X)=1 .
$$

REMARK. If $E$ is a countable set, $\mathbb{P}$ a Gibbs measure on $\operatorname{Conf}(E)$ corresponding to the Hamiltonian $H$ of pairwise interaction of particles (cf. e.g., Sinai [29]), $p, q$ are points in $E$ and $\sigma_{p q}$ the transposition of $p$ and $q$, then, for almost every configuration $X$, conditioned to contain a particle at $q$ but not at $p$, by definition, we have

$$
\frac{d \mathbb{P} \circ \sigma_{p q}}{d \mathbb{P}}(X)=\prod_{x \in X: x \neq q} \exp (H(p, x)-H(q, x)) .
$$

The quasi-invariance property established in this paper is thus, informally speaking, the analogue of the Gibbs property, with Hamiltonian $H(x, y)=2 \log |x-y|$, for determinantal point processes.

1.6. Examples of regularized multiplicative functionals. Regularization of a multiplicative functional can take different form depending on the specific process. We illustrate this by two examples.

The sine-process. The argument below is valid for the continuous sine-process as well as the discrete sine-process. The sine-process is stationary, therefore, for almost every configuration $X$ the series

$$
\sum_{x \in X: x \neq 0} \frac{1}{x}
$$


diverges absolutely since so does the harmonic series. Nonetheless, the series (16) converges conditionally in principal value: the limit

$$
\lim _{N \rightarrow \infty} \sum_{x \in X: x \neq 0,|x| \leq N} \frac{1}{x}
$$

exists in $L_{2}$ and almost surely along a subsequence, e.g., $N^{4}$. Similarly, for distinct points $p_{1}, \ldots, p_{l}, q_{1}, \ldots, q_{l}$, taken in $\mathbb{R}$ in the continuous case and in $\mathbb{Z}$ in the discrete case, the limit

$$
\lim _{N \rightarrow \infty} \prod_{x \in X,|x| \leq N, x \neq q_{1}, \ldots, q_{l}} \prod_{i=1}^{l}\left(\frac{x-p_{i}}{x-q_{i}}\right)^{2},
$$

exists and has finite expectation. The normalized mutliplicative functional is in this case precisely the limit (17) normalized to have expectation 1.

The determinantal point process with the Gamma-kernel. The determinantal point process with the Gamma-kernel, introduced by Borodin and Olshanski in [3] and for which the quasi-invariance under the action of the infinite symmetric group is due to Olshanski [22], is a point process on the phase space $\mathbb{Z}^{\prime}=1 / 2+\mathbb{Z}$ of half-integers such that for almost every configuration $X$ we have

$$
\sum_{x \in X: x>0} \frac{1}{x}<+\infty, \quad \sum_{y \notin X: y<0} \frac{1}{|y|}<+\infty .
$$

Furthermore, each sum in (18), considered as a random variable on the space of configurations on $\mathbb{Z}^{\prime}$, has finite variance with respect to the determinantal point process with the Gamma-kernel.

For $p, q \in \mathbb{Z}^{\prime}$, the normalized multiplicative functional corresponding to the function $g(x)=((x-p) /(x-q))^{2}$ will therefore have the form

$$
C \cdot \prod_{x \in X, x>0} g(x) \cdot \prod_{y \notin X: y<0} g^{-1}(y),
$$

where the constant $C$ is chosen in such a way that the resulting expression have expectation 1 .

1.7. Outline of the argument. We start with the discrete case and illustrate the argument in the specific case of a transposition of two distinct points $p, q \in E$. A theorem due to Lyons [19], Shirai-Takahashi [27] states that the measure $\mathbb{P}_{\Pi}^{p, \breve{q}}$ is the conditional measure of $\mathbb{P}_{\Pi}$ on the subset of configurations containing a particle at $p$ and not containing a particle at $q$.

Step 1. The Relation Between Palm Subspaces. The key point in the proof of Proposition 1.8 is the equality

$$
L(p, \breve{q})=\frac{x-p}{x-q} L(q, \breve{p}),
$$


which it is more convenient to rewrite in the form

$$
L(p, \breve{q})=\chi_{E \backslash\{p, q\}} \frac{x-p}{x-q} L(q, \breve{p}) .
$$

The equality (20) directly follows from the integrability of the discrete sinekernel. The remainder of the argument shows that the relation (20) implies the relation

$$
\mathbb{P}_{\Pi}^{p, \breve{q}}=\bar{\Psi}(p, q) \mathbb{P}_{\Pi}^{q, \breve{p}},
$$

which, in turn, is a reformulation of Proposition 1.8.

Step 2. Multiplicative functionals of determinantal point processes. Given a function $g$ on $\mathbb{Z}$, the multiplicative functional $\Psi_{g}$ is defined on $\operatorname{Conf}(E)$ by the formula

$$
\Psi_{g}(X)=\prod_{x \in X} g(x)
$$

provided that the infinite product in the right-hand side converges absolutely.

At the centre of the argument lies the result of [5] that can informally be summarized as follows: a determinantal measure times a multiplicative functional is, after normalization, again a determinantal measure. More precisely, let $g$ be a positive function on $E$ bounded away from 0 and $\infty$, and let $\Pi$ be an operator of orthogonal projection in $L_{2}(E)$ onto a closed subspace $L$. Let $\Pi^{g}$ be the operator of orthogonal projection onto the subspace $\sqrt{g} L$. Then, under certain additional assumptions we have

$$
\mathbb{P}_{\Pi^{g}}=\frac{\Psi_{g} \mathbb{P}_{\Pi}}{\int_{\operatorname{Conf}(E)} \Psi_{g} d \mathbb{P}_{\Pi}} .
$$

The relation (22) together with the relation (20) suggests that the measures $\mathbb{P}_{\Pi}^{p, \breve{q}}$ and $\mathbb{P}_{\Pi}^{q, \breve{p}}$ are equivalent, and the Radon-Nikodym derivative is given by the normalized multiplicative functional corresponding to the function

$$
g^{p, \breve{q}}(x)=\frac{x-p}{x-q} \chi_{E \backslash\{p, q\}} .
$$

Step 3. Regularization of multiplicative functionals. A technical difficulty arises that in many examples the multiplicative functional corresponding to the function $g^{p, \breve{q}}$ fails to converge absolutely with respect to the measure $\mathbb{P}_{\Pi}^{p, \breve{q}}$; indeed, in many examples (in particular, for stationary determinantal processes on $\mathbb{Z}$ ), we have

$$
\sum_{x \in E}\left|g^{p, \breve{q}}(x)-1\right|=+\infty
$$

and, consequently, also

$$
\sum_{x \in E}\left|g^{p, \breve{q}}(x)-1\right| \cdot \Pi^{p, \breve{q}}(x, x)=+\infty .
$$


In order to resolve this difficulty, we go back to the formula (22). For multiplicative functional $\Psi_{g}$ integrable with respect to a determinantal measure $\mathbb{P}_{\Pi}$ set

$$
\bar{\Psi}_{g}=\frac{\Psi_{g}}{\int \Psi_{g} d \mathbb{P}_{\Pi}} .
$$

The functional $\bar{\Psi}_{g}$ will be called the normalized multiplicative functional corresponding to $\Psi_{g}$ and $\mathbb{P}_{\Pi}$. To keep notation lighter, we do not explicitly indicate dependence on $\Pi$; in what follows, the precise measure, with respect to which normalization is taken, will be clear from the context. We now rewrite (22) in the form

$$
\mathbb{P}_{\Pi g}=\bar{\Psi}_{g} \cdot \mathbb{P}_{\Pi} \cdot
$$

The key observation for the remainder of the argument is that the definition of the normalized multiplicative functional $\bar{\Psi}_{g}$ can be extended in such a way that (24) continues to hold for a wider class of functions $g$, for which the multiplicative functional itself diverges almost surely.

We first explain the idea of this extension for additive functionals. Given a measurable function $f$ on $E$, the corresponding additive functional on $\operatorname{Conf}(E)$ is defined by the formula

$$
S_{f}(X)=\sum_{x \in X} f(x)
$$

provided the series in the right hand side converges absolutely. The expectation of the additive functional with respect to $\mathbb{P}_{\Pi}$ is given by the formula

$$
\mathbb{E}_{\mathbb{P}_{\Pi}} S_{f}=\sum_{x \in E} f(x) \Pi(x, x),
$$

provided, again, that the series in the right-hand side converges absolutely. For the variance of the additive functional, we have

$$
\operatorname{Var}_{\mathbb{P}_{\Pi}} S_{f}=\frac{1}{2} \sum_{x, y \in E}(f(x)-f(y))^{2}(\Pi(x, y))^{2} .
$$

Let

$$
\bar{S}_{f}=S_{f}-\mathbb{E}_{\mathbb{P}_{\Pi}} S_{f}
$$

be the normalized additive functional corresponding to the function $f$. It is easy to give examples of functions $f$ for which the sum in the right-hand side of (25) diverges while the sum in the right-hand side of (26) converges. For such functions, convergence of the sum in the right-hand side of (26) allows one to define the normalized additive functional $\bar{S}_{f}$ by continuity, even though the additive functional $S_{f}$ itself is not defined. In a similar way, for a function $g$ bounded away from 0 and $\infty$ and satisfying

$$
\sum_{x \in E}|g(x)-1|^{2} \Pi(x, x)<+\infty
$$


one can define the normalized multiplicative functional $\bar{\Psi}_{g}$, even though the multiplicative functional $\Psi_{g}$ itself need not be defined; the relation (24) still holds.

We thus check that the normalized multiplicative functional $\bar{\Psi}_{g p, q}$ can be defined with respect to the measure $\mathbb{P}_{\Pi}^{q, \breve{p}}$; the relation (20) now implies the desired equality (21). This completes the outline of the proof of Theorem 1.6.

The proof in continuous case follows a similar scheme. The rôle of conditional measures is played by reduced Palm measures. The reduced Palm measure $\mathbb{P}_{\Pi}^{q_{1}, \ldots, q_{l}}$ of the measure $\mathbb{P}_{\Pi}$ with respect to $l$ distinct points $q_{1}, \ldots, q_{l} \in \mathbb{R}$ is the determinantal measure corresponding to the operator $\Pi^{q_{1}, \ldots, q_{l}}$ of the orthogonal projection onto the subspace

$$
L\left(q_{1}, \ldots, q_{l}\right)=\left\{\varphi \in L: \varphi\left(q_{1}\right)=\cdots=\varphi\left(q_{l}\right)=0\right\} .
$$

The continuous analogue of the equality (20) is the relation

$$
L\left(p_{1}, \ldots, p_{l}\right)=\frac{\left(x-p_{1}\right) \cdots\left(x-p_{l}\right)}{\left(x-q_{1}\right) \cdots\left(x-q_{l}\right)} L\left(q_{1}, \ldots, q_{l}\right)
$$

valid for $\mu^{\otimes l}$-almost any two $l$-tuples of distinct points $\left(p_{1}, \ldots, p_{l}\right),\left(q_{1}, \ldots, q_{l}\right)$ in $\mathbb{R}$.

The next step is to regularize the multiplicative functional corresponding to the function

$$
\frac{\left(x-p_{1}\right) \cdots\left(x-p_{l}\right)}{\left(x-q_{1}\right) \cdots\left(x-q_{l}\right)}
$$

while the overall scheme of regularization is the same as in the discrete case, additional estimates are needed here because the function (28) is bounded away neither from zero nor from infinity.

The resulting normalized multiplicative functional $\bar{\Psi}\left(p_{1}, \ldots, p_{l}, q_{1}, \ldots, q_{l}\right)$ is then seen to be the Radon-Nikodym derivative of the reduced Palm measures $\mathbb{P}_{\Pi}^{p_{1}, \ldots, p_{l}}$ and $\mathbb{P}_{\Pi}^{q_{1}, \ldots, q_{l}}$, which, in turn, implies Theorem 1.4.

1.8. Organization of the paper. The paper is organized as follows. In Section 2, we collect necessary facts about determinantal point processes, their multiplicative functionals and their Palm measures. We recall the results of [5] (see also [7]) showing that the product of a determinantal measure with a multiplicative functional is, after normalization, again a determinantal measure, whose kernel is found explicitly. We also check that equivalence of reduced Palm measures corresponding to distinct $l$-tuples of points implies the quasi-invariance of the point process under Borel automorphisms preserving the class of its correlation measures and acting by the identity beyond a bounded set. In Section 3, we start by showing that reduced Palm measures of determinantal point processes given by projection operators with integrable kernels are themselves determinantal point processes given by projection operators with integrable kernels and proceed to verify the key relations (51) and (52) showing that the ranges of projection operators 
corresponding to reduced Palm measures at distinct points differ by multiplication by a function.

Proposition 4.2 in Section 4 describes the regularization of multiplicative functionals. Relations (51) and (52) are then seen to imply that the reduced Palm measures themselves are equivalent, and that the corresponding Radon-Nikodym derivative is a regularized multiplicative functional. This completes the proof of Theorems 1.4 and 1.6.

REMARK. After this work was completed, I became aware of the preprint $A b$ solute continuity and singularity of Palm measures of the Ginibre point process, arXiv:1406.3913, by Hirofumi Osada and Tomoyuki Shirai, in which, for the Ginibre ensemble, using its finite-dimensional approximations by orthogonal polynomial ensembles, the authors establish the equivalence of reduced Palm measures, conditioned at distinct $l$-tuples of points in $\mathbb{C}$, and represent the Radon-Nikodym derivative as a regularized multiplicative functional.

\section{Point processes and Palm distributions.}

2.1. Spaces of configurations. Let $E$ be a locally compact complete metric space. A configuration on $E$ is a collection of points in $E$, called particles, considered without regard to order and subject to the additional requirement that every bounded set contain only finitely many particles of a configuration (cf. Lenard [18], Daley-Vere-Jones [10]). Let $\operatorname{Conf}(E)$ be the space of configurations on $E$. To a configuration $X \in \operatorname{Conf}(E)$ assign a Radon measure $\sum_{x \in X} \delta_{x}$ on the space $E$; this correspondence identifies the space $\operatorname{Conf}(E)$ with the space of integer-valued Radon measures on $E$. The space $\operatorname{Conf}(E)$ is thus endowed with a natural structure of a complete separable metric space. The Borel structure on the space $\operatorname{Conf}(E)$ can equivalently be defined without introducing a topology explicitly: namely, for a bounded Borel set $B \subset E$, let

$$
\#_{B}: \operatorname{Conf}(E) \rightarrow \mathbb{N} \cup\{0\}
$$

be the function that to a configuration assigns the number of its particles belonging to $B$. The random variables $\#_{B}$ over all bounded Borel sets $B \subset E$ determine the Borel sigma-algebra on Conf $(E)$, cf. Daley-Vere-Jones [10], Kolmogoroff [17].

2.2. Multiplicative functionals. We next recall the definition of multiplicative functionals on spaces of configurations. Let $g$ be a nonnegative measurable function on $E$, and introduce the multiplicative functional $\Psi_{g}: \operatorname{Conf}(E) \rightarrow \mathbb{R}$ by the formula

$$
\Psi_{g}(X)=\prod_{x \in X} g(x)
$$

If the infinite product $\prod_{x \in X} g(x)$ absolutely converges to 0 or to $\infty$, then we set, respectively, $\Psi_{g}(X)=0$ or $\Psi_{g}(X)=\infty$. If the product in the right-hand side fails to converge absolutely, then the multiplicative functional is not defined. 
2.3. Point processes. A Borel probability measure $\mathbb{P}$ on $\operatorname{Conf}(E)$ is called $a$ point process with phase space $E$.

We recall that the process $\mathbb{P}$ is said to admit correlation functions of order $l$ if for any continuous compactly supported function $f$ on $E^{l}$ the functional

$$
\sum_{x_{1}, \ldots, x_{l} \in X} f\left(x_{1}, \ldots, x_{l}\right)
$$

is $\mathbb{P}$-integrable; here the sum is taken over all $l$-tuples of distinct particles in $X$. The $l$ th correlation measure $\rho_{l}$ of the point process $\mathbb{P}$ is then defined by the formula

$$
\mathbb{E}_{\mathbb{P}}\left(\sum_{x_{1}, \ldots, x_{l} \in X} f\left(x_{1}, \ldots, x_{l}\right)\right)=\int_{E^{l}} f\left(q_{1}, \ldots, q_{l}\right) d \rho_{l}\left(q_{1}, \ldots, q_{l}\right) .
$$

By definition, a point process $\mathbb{P}$ is uniquely determined by prescribing joint distributions, with respect to $\mathbb{P}$, of random variables $\#_{B_{1}}, \ldots, \#_{B_{l}}$ over all finite collections of disjoint bounded Borel subsets $B_{1}, \ldots, B_{l} \subset E$. Since, for arbitrary nonzero complex numbers $z_{1}, \ldots, z_{l}$ inside the unit circle, the function $\prod_{k=1}^{l} z_{k}^{\#_{B_{k}}}$ is a well-defined multiplicative functional on $\operatorname{Conf}(E)$, that, moreover, takes values inside the unit circle, a point process $\mathbb{P}$ on $\operatorname{Conf}(E)$ is also uniquely determined by prescribing the values of expectations of multiplicative functionals of this form.

2.4. Campbell measures. Following Kallenberg [16] and Daley-Vere-Jones [10], we now recall the definition of Campbell measures of point processes.

Take a Borel probability measure $\mathbb{P}$ on $\operatorname{Conf}(E)$ of finite local intensity, that is, admitting the first correlation measure $\rho_{1}$, or, equivalently, such that for any bounded Borel set $B$, the function $\#_{B}$ is integrable with respect to $\mathbb{P}$. For any bounded Borel set $B \subset E$, by definition we then have

$$
\rho_{1}(B)=\int_{\operatorname{Conf}(E)} \#_{B}(X) d \mathbb{P}(X) .
$$

The Campbell measure $\mathcal{C}_{\mathbb{P}}$ of a Borel probability measure $\mathbb{P}$ of finite local intensity on $\operatorname{Conf}(E)$ is a sigma-finite measure on $E \times \operatorname{Conf}(E)$ such that for any Borel subsets $B \subset E, \mathscr{Z} \subset \operatorname{Conf}(E)$ we have

$$
\mathcal{C}_{\mathbb{P}}(B \times \mathscr{Z})=\int_{\mathscr{Z}} \#_{B}(X) d \mathbb{P}(X) .
$$

For a point process admitting correlation functions of order $l$ one can also define the $l$ th iterated Campbell measure $\mathcal{C}_{\mathbb{P}}^{l}$ of the point process $\mathbb{P}$, that is, by definition, a measure on $E^{l} \times \operatorname{Conf}(E)$ such that for any disjoint bounded sets $B_{1}, \ldots, B_{l} \subset E$ and any measurable subset $\mathscr{Z} \subset \operatorname{Conf}(E)$ we have

$$
\mathrm{C}_{\mathbb{P}}^{l}\left(B_{1} \times \cdots \times B_{l} \times \mathscr{Z}\right)=\int_{\mathscr{Z}} \#_{B_{1}}(X) \times \cdots \times \#_{B_{l}}(X) d \mathbb{P}(X) .
$$


2.5. Palm distributions. Following Kallenberg [16] and Daley-Vere-Jones [10], we now recall the construction of Palm distributions from Campbell measures. For a fixed Borel $\mathscr{Z} \subset \operatorname{Conf}(E)$ the Campbell measure $\mathcal{C}_{\mathbb{P}}$ induces a sigmafinite measure $\mathcal{C}_{\mathbb{P}}^{\mathscr{Z}}$ on $E$ by the formula

$$
\mathcal{C}_{\mathbb{P}}^{\mathscr{Z}}(B)=\mathcal{C}_{\mathbb{P}}(B \times \mathscr{Z}) .
$$

By definition, for any Borel subset $\mathscr{Z} \subset \operatorname{Conf}(E)$ the measure $\mathcal{C}_{\mathbb{P}}^{\mathscr{Z}}$ is absolutely continuous with respect to $\rho_{1}$. We now take $q \in E$, vary $\mathscr{Z}$ and set

$$
\hat{\mathbb{P}}^{q}(\mathscr{Z})=\frac{d \mathcal{C}_{\mathbb{P}}^{\mathscr{Z}}}{d \rho_{1}}(q) .
$$

For $\rho_{1}$-almost every $q \in E$, the expression $\hat{\mathbb{P}}^{q}(\mathscr{Z})$, considered as a function of $\mathscr{Z}$ (while $q$ stays fixed), defines a probability measure $\hat{\mathbb{P}}^{q}$ on $\operatorname{Conf}(E)$, the Palm measure of $\mathbb{P}$ at the point $q$. Equivalently, the Palm measure $\hat{\mathbb{P}}^{q}$ is the canonical conditional measure, in the sense of Rohlin [24], of the Campbell measure $\mathcal{C}_{\mathbb{P}}$ with respect to the measurable partition of the space $E \times \operatorname{Conf}(E)$ into subsets of the form $\{q\} \times \operatorname{Conf}(E), q \in E$.

Similarly, using iterated Campbell measures one defines iterated Palm measures: for a fixed Borel $\mathscr{Z} \subset \operatorname{Conf}(E)$ the $l$ th iterated Campbell measure $\mathcal{C}_{\mathbb{P}}^{l}$ induces a sigma-finite measure $\mathrm{e}_{\mathbb{P}}^{l, \mathscr{Z}}$ on $E$ by the formula

$$
\mathrm{C}_{\mathbb{P}}^{l, \mathscr{Z}}(B)=\mathcal{C}_{\mathbb{P}}(B \times \mathscr{Z}) .
$$

By definition, for any Borel subset $\mathscr{Z} \subset \operatorname{Conf}(E)$ the measure $\mathcal{C}_{\mathbb{P}}^{l, \mathscr{Z}}$ is absolutely continuous with respect to the $l$ th correlation measure $\rho_{l}$ of our point process $\mathbb{P}$. For $\rho_{l}$-almost all $\left(q_{1}, \ldots, q_{l}\right) \in E^{l}$, one can therefore define a probability measure $\hat{\mathbb{P}}^{q_{1}}, \ldots, q_{l}$ on $\operatorname{Conf}(E)$ by the formula

$$
\hat{\mathbb{P}}^{q_{1}, \ldots, q_{l}}(\mathscr{Z})=\frac{d \mathcal{C}_{\mathbb{P}}^{l, \mathscr{Z}}}{d \rho_{1}}\left(q_{1}, \ldots, q_{l}\right) .
$$

The measure $\hat{\mathbb{P}}^{q_{1}}, \ldots, q_{l}$ is called the $l$ th iterated Palm measure of the point process $\mathbb{P}$. The iterated Palm measure $\hat{\mathbb{P}}^{q}$ is the canonical conditional measure, in the sense of Rohlin [24], of the Campbell measure $\mathcal{C}_{\mathbb{P}}^{l}$ with respect to the measurable partition of the space $E^{l} \times \operatorname{Conf}(E)$ into subsets of the form $\left\{q_{1}, \ldots, q_{l}\right\} \times \operatorname{Conf}(E)$, with $q_{1}, \ldots, q_{l} \in E$ distinct.

For distinct points $q_{1}, \ldots, q_{l}$, the $l$ th iterated Palm measure of course satisfies

$$
\hat{\mathbb{P}}^{q_{1}, \ldots, q_{l}}=\left(\cdots\left(\hat{\mathbb{P}}^{q_{1}}\right)^{q_{2}} \ldots\right)^{q_{l}} .
$$




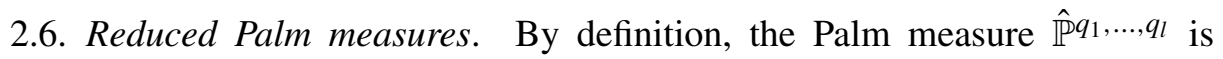
supported on the subset of configurations containing a particle at each position $q_{1}, \ldots, q_{l}$. It is often convenient to remove these particles and to define the reduced Palm measure $\mathbb{P}^{q_{1}, \ldots, q_{l}}$ as the push-forward of the Palm measure $\hat{\mathbb{P}}^{q_{1}, \ldots, q_{l}}$ under the erasing map $X \rightarrow X \backslash\left\{q_{1}, \ldots, q_{l}\right\}$. Reduced Palm measures allow one to give a convenient representation for measures of cylinder sets. Take $X_{0} \in \operatorname{Conf}(E)$ and $q_{1}^{(0)}, \ldots, q_{l}^{(0)} \in X_{0}$.

Take disjoint bounded open sets $B^{(1)}, \ldots, B^{(l)} \subset E$ such that $q_{i}^{(0)} \in B^{(i)}, q_{i}^{(0)}=$ $X_{0} \cap \bar{B}^{(i)}$ for all $i=1, \ldots, l$. Set $B=\cup B^{(i)}$ and take an open set $V \subset E$ with bounded complement, disjoint from all $B^{(i)}$, and satisfying $X_{0} \backslash\left\{q_{1}^{(0)}, \ldots, q_{l}^{(0)}\right\} \subset$ $V$. Let $\mathscr{W}$ be a neighbourhood of $X_{0} \backslash\left\{q_{1}^{(0)}, \ldots, q_{l}^{(0)}\right\}$ in $\operatorname{Conf}(E)$ satisfying

$$
\mathscr{W} \subset\{X \in \operatorname{Conf}(E): X \subset V\} .
$$

Introduce a neighbourhood $\mathscr{Z}$ of $X_{0}$ by setting

$$
\mathscr{Z}=\left\{X \in \operatorname{Conf}(E): \#_{B^{(1)}}(X)=\cdots=\#_{B^{(l)}}(X)=1,\left.X\right|_{E \backslash B} \subset \mathscr{W}\right\} .
$$

Proposition 2.1. We have

$$
\mathbb{P}(\mathscr{Z})=\int_{B^{(1)} \times \cdots \times B^{(l)}} \mathbb{P}^{q_{1}, \ldots, q_{l}}(\mathscr{W}) d \rho_{l}\left(q_{1}, \ldots, q_{l}\right) .
$$

Proof. We disintegrate $\mathcal{C}^{(l)}\left(B^{(1)} \times \cdots \times B^{(l)} \times \mathscr{Z}\right)$ in two ways. By definition of iterated Palm measures, we have

$$
\mathrm{\varrho}^{(l)}\left(B^{(1)} \times \cdots \times B^{(l)} \times \mathscr{Z}\right)=\int_{B^{(1)} \times \cdots \times B^{(l)}} \hat{\mathbb{P}}^{q_{1}, \ldots, q_{l}}(\mathscr{Z}) d \rho_{l}\left(q_{1}, \ldots, q_{l}\right) .
$$

By definition of iterated Campbell measures, see (30), (31), we have

$$
\mathrm{e}^{(l)}\left(B^{(1)} \times \cdots \times B^{(l)} \times \mathscr{Z}\right)=\int_{\mathscr{Z}} \#_{B^{(1)}}(X) \times \cdots \times \#_{B^{(l)}}(X) d \mathbb{P}(X)=\mathbb{P}(\mathscr{Z}) .
$$

Passing to reduced Palm measures gives $\hat{\mathbb{P}}^{q_{1}, \ldots, q_{l}}(\mathscr{Z})=\mathbb{P}^{q_{1}, \ldots, q_{l}}(\mathscr{W})$, and (31) follows.

2.7. Locally trace class operators and their kernels. Let $\mu$ be a sigma-finite Borel measure on $E$. The inner product in $L_{2}(E, \mu)$ is always denoted by the symbol $\langle$,$\rangle .$

Let $\mathscr{I}_{1}(E, \mu)$ be the ideal of trace class operators $\widetilde{K}: L_{2}(E, \mu) \rightarrow L_{2}(E, \mu)$ (see [23] for the precise definition); the symbol $\|\widetilde{K}\|_{\mathscr{I}_{1}}$ will stand for the $\mathscr{I}_{1}$ norm of the operator $\widetilde{K}$. Let $\mathscr{I}_{2}(E, \mu)$ be the ideal of Hilbert-Schmidt operators $\widetilde{K}: L_{2}(E, \mu) \rightarrow L_{2}(E, \mu)$; the symbol $\|\widetilde{K}\|_{\mathscr{I}_{2}}$ will stand for the $\mathscr{I}_{2}$-norm of the operator $\widetilde{K}$. 
Let $\mathscr{I}_{1, \text { loc }}(E, \mu)$ be the space of operators $K: L_{2}(E, \mu) \rightarrow L_{2}(E, \mu)$ such that for any bounded Borel subset $B \subset E$ we have

$$
\chi_{B} K \chi_{B} \in \mathscr{I}_{1}(E, \mu) \text {. }
$$

Again, we endow the space $\mathscr{I}_{1, \text { loc }}(E, \mu)$ with a countable family of semi-norms

$$
\left\|\chi_{B} K \chi_{B}\right\|_{\mathscr{I}_{1}},
$$

where, as before, $B$ runs through an exhausting family $B_{n}$ of bounded sets. A locally trace class operator $K$ admits a kernel, for which, slightly abusing notation, we use the same symbol $K$.

2.8. Determinantal point processes. A Borel probability measure $\mathbb{P}$ on $\operatorname{Conf}(E)$ is called determinantal if there exists an operator $K \in \mathscr{I}_{1, \text { loc }}(E, \mu)$ such that for any bounded measurable function $g$, for which $g-1$ is supported in a bounded set $B$, we have

$$
\mathbb{E}_{\mathbb{P}} \Psi_{g}=\operatorname{det}\left(1+(g-1) K \chi_{B}\right) .
$$

Here and elsewhere in similar formulas, 1 stands for the identity operator. The Fredholm determinant in (33) is well defined since $K \in \mathscr{I}_{1, \mathrm{loc}}(E, \mu)$. The equation (33) determines the measure $\mathbb{P}$ uniquely. For any pairwise disjoint bounded Borel sets $B_{1}, \ldots, B_{l} \subset E$ and any $z_{1}, \ldots, z_{l} \in \mathbb{C}$ from (33), we have $\mathbb{E}_{\mathbb{P}} z_{1}^{\#_{B_{1}}} \cdots z_{l}{ }^{\#_{B_{l}}}=$ $\operatorname{det}\left(1+\sum_{j=1}^{l}\left(z_{j}-1\right) \chi_{B_{j}} K \chi_{\sqcup_{i} B_{i}}\right)$.

For further results and background on determinantal point processes, see, for example, [12, 14, 19, 20, 26, 27, 30].

If $K$ belongs to $\mathscr{I}_{1, \text { loc }}(E, \mu)$, then, throughout the paper, we denote the corresponding determinantal measure by $\mathbb{P}_{K}$. Note that $\mathbb{P}_{K}$ is uniquely defined by $K$, but different operators may yield the same measure. By a theorem due to Macchì and Soshnikov [21, 30] and Shirai-Takahashi [25], any Hermitian positive contraction that belongs to the class $\mathscr{I}_{1, \text { loc }}(E, \mu)$ defines a determinantal point process. For the purposes of this paper, we will only be interested in determinantal point processes given by operators of orthogonal projection; in the case of a discrete phase space, there is a standard procedure of doubling the phase space (see, e.g., [19]) that reduces the case of contractions to the case of projections.

2.9. Weak convergence in the space of configurations. The space $\operatorname{Conf}(E)$ is endowed with a natural structure of a complete separable metric space, and the space of finite Borel measures on the space of configurations is consequently also a complete separable metric space with respect to the weak topology. If $\varphi: E \rightarrow \mathbb{R}$ be a compactly supported continuous function, then a measurable function $\#_{\varphi}: \operatorname{Conf}(E) \rightarrow \mathbb{R}$ is introduced by the formula $\#_{\varphi}(X)=\sum_{x \in X} \varphi(x)$. Theorem 11.1.VII in [10] states that if $\mathbb{P}_{n}, n \in \mathbb{N}$ and $\mathbb{P}$ are Borel probability measures on $\operatorname{Conf}(E)$, then the measures $\mathbb{P}_{n}$ converge to $\mathbb{P}$ weakly as $n \rightarrow \infty$ if and only if 
for any finite collection $\varphi_{1}, \ldots, \varphi_{l}$ of continuous functions with disjoint compact supports the joint distributions of the random variables $\#_{\varphi_{1}}, \ldots, \#_{\varphi_{l}}$ with respect to $\mathbb{P}_{n}$ converge, as $n \rightarrow \infty$, to the joint distribution of $\#_{\varphi_{1}}, \ldots, \#_{\varphi_{l}}$ with respect to $\mathbb{P}$; convergence of joint distributions being understood according to the weak topology on the space of Borel probability measures on $\mathbb{R}^{l}$. From the definition of determinantal point processes, we now have:

Proposition 2.2. Let $K_{n}, n \in \mathbb{N}, K$ be locally trace class operators acting in $L_{2}(E, \mu)$ and inducing determinantal measures $\mathbb{P}_{K_{n}}, \mathbb{P}_{K}$. If $K_{n} \rightarrow K$ in $\mathscr{I}_{1, \text { loc }}(E, \mu)$ as $n \rightarrow \infty$, then $\mathbb{P}_{K_{n}} \rightarrow \mathbb{P}_{K}$ weakly in the space of probability measures on $\operatorname{Conf}(E)$.

2.10. The product of a determinantal measure and a multiplicative functional. We start by recalling the results of $[5,7]$ showing that the product of a determinantal measure with a multiplicative functional is, after normalization, again a determinantal measure, whose kernel is found explicitly.

Let $g$ be a nonnegative measurable function on $E$. If the operator $1+(g-1) K$ is invertible, then we set

$$
\mathfrak{B}(g, K)=g K(1+(g-1) K)^{-1}, \quad \tilde{\mathfrak{B}}(g, K)=\sqrt{g} K(1+(g-1) K)^{-1} \sqrt{g} .
$$

By definition, $\mathfrak{B}(g, K), \widetilde{\mathfrak{B}}(g, K) \in \mathscr{I}_{1, \mathrm{loc}}(E, \mu)$ since $K \in \mathscr{I}_{1, \mathrm{loc}}(E, \mu)$, and, if $K$ is self-adjoint, then so is $\widetilde{\mathfrak{B}}(g, K)$ [this follows from the elementary identity $p(1-q p)^{-1}=(1-p q)^{-1} p$ that holds for arbitrary bounded operators $p, q$ provided both sides are well defined].

We now quote Proposition 2.1 in [7].

Proposition 2.3. Let $K \in \mathscr{I}_{1, \mathrm{loc}}(E, \mu)$ be a self-adjoint positive contraction, and let $\mathbb{P}_{K}$ be the corresponding determinantal measure on $\operatorname{Conf}(E)$. Let $g$ be a nonnegative bounded measurable function on $E$ such that

$$
\sqrt{g-1} K \sqrt{g-1} \in \mathscr{I}_{1}(E, \mu)
$$

and that the operator $1+(g-1) K$ is invertible. Then the operators $\mathfrak{B}(g, K)$, $\widetilde{\mathfrak{B}}(g, K)$ induce on $\operatorname{Conf}(E)$ a determinantal measure $\mathbb{P}_{\mathfrak{B}(g, K)}=\mathbb{P}_{\widetilde{\mathfrak{B}}_{(g, K)}}$ satisfying

$$
\mathbb{P}_{\mathfrak{B}(g, K)}=\frac{\Psi_{g} \mathbb{P}_{K}}{\int_{\operatorname{Conf}(E)} \Psi_{g} d \mathbb{P}_{K}}
$$

REMARK. Here and elsewhere, we write $\sqrt{g-1} K \sqrt{g-1}$ instead of the longer and more formal $\operatorname{sgn}(g-1) \sqrt{|g-1|} K \sqrt{|g-1|}$. 
REMARK. Of course, from (34) and the invertibility of the operator $1+$ $(g-1) K$ we have $\Psi_{g} \in L_{1}\left(\operatorname{Conf}(E), \mathbb{P}_{K}\right)$ and

$$
\int \Psi_{g} d \mathbb{P}_{K}=\operatorname{det}(1+\sqrt{g-1} K \sqrt{g-1})>0,
$$

so the right-hand side of (35) is well defined.

For the reader's convenience, we recall the proof of Proposition 2.3 in the case when the assumption (34) is replaced (cf. [5]) by a simpler assumption

$$
(g-1) K \in \mathscr{I}_{1}(E, \mu)
$$

for the general case, see the proof of Proposition 2.1 in [7]. Take a bounded measurable function $f$ on $E$ such that $(f-1) K \in \mathscr{I}_{1}(E, \mu)$; for example, one may take $f$ that is different from 1 on a bounded set. We have $(f g-1) K \in \mathscr{I}_{1}(E, \mu)$ since $(f-1) K \in \mathscr{I}_{1}(E, \mu),(g-1) K \in \mathscr{I}_{1}(E, \mu)$. By definition, we have

$$
\begin{aligned}
\mathbb{E}_{\mathbb{P}_{K}} \Psi_{f} \Psi_{g} & =\operatorname{det}(1+(f g-1) K) \\
& =\operatorname{det}\left(1+(f-1) g K(1+(g-1) K)^{-1}\right) \operatorname{det}(1+(g-1) K) .
\end{aligned}
$$

We rewrite (36) in the form

$$
\frac{\mathbb{E}_{\mathbb{P}_{K}} \Psi_{f} \Psi_{g}}{\mathbb{E}_{\mathbb{P}_{K}} \Psi_{g}}=\operatorname{det}(1+(f-1) \mathfrak{B}(g, K))=\operatorname{det}(1+(f-1) \widetilde{\mathfrak{B}}(g, K)) .
$$

Since a probability measure on the space of configurations is uniquely determined by the values of multiplicative functionals corresponding to all bounded functions $f$ that are different from 1 on a bounded set, formula (36) implies Proposition 2.3.

2.11. Projections and subspaces. Let $L \subset L_{2}(E, \mu)$ be a closed subspace, let $\Pi$ be the corresponding projection operator, assumed to be locally of trace class, and let $\mathbb{P}_{\Pi}$ the corresponding determinantal measure. Our aim is to determine how the measure $\mathbb{P}_{\Pi}$ changes if the subspace $L$ is multiplied by a function. We start with the following clear proposition.

PROPOSITION 2.4. Let $\alpha(x)$ be a measurable function such that $|\alpha(x)|=1$ $\mu$-almost surely. Then the operator of orthogonal projection onto the subspace $\alpha(x) L$ induces the same determinantal measure $\mathbb{P}_{\Pi}$.

PROOF. Indeed, if $\Pi(x, y)$ is the kernel of the operator $\Pi$, then the kernel of the new operator has the form

$$
\frac{\alpha(x) \Pi(x, y)}{\alpha(y)},
$$

and such gauge transformations do not change the determinantal measure. 
PROPOSITION 2.5. Let $g$ be a nonnegative bounded function on E such that the operator $1+(g-1) \Pi$ is invertible. Then the operator

$$
\Pi^{g}=\sqrt{g} \Pi(1+(g-1) \Pi)^{-1} \sqrt{g}
$$

is the operator of orthogonal projection onto the closure of the subspace $\sqrt{g} L$.

Proof. First, let $\widetilde{\varphi} \in \sqrt{g} L$, that is, $\widetilde{\varphi}=\sqrt{g} \varphi, \varphi \in L$. Since $\varphi \in L$, we have

$$
(1+(g-1) \Pi) \varphi=g \varphi,
$$

whence

$$
(1+(g-1) \Pi)^{-1} \sqrt{g} \widetilde{\varphi}=\varphi
$$

and finally

$$
\Pi^{g} \widetilde{\varphi}=\widetilde{\varphi}
$$

as desired.

Now take $\varphi$ to be orthogonal to the subspace $\sqrt{g} L$. Since $g$ is real-valued, we have $\sqrt{g} \varphi \in L^{\perp}$, whence $(1+(g-1) \Pi) \varphi=\sqrt{g} \varphi$ and, consequently, $(1+(g-$ 1) $\Pi)^{-1} \sqrt{g} \varphi=\varphi$. We thus have $\Pi^{g} \varphi=0$, and the proposition is proved completely.

We prepare a useful proposition for proving local trace-class convergence of sequences of operators $\Pi^{g}$.

Proposition 2.6. Let $g_{n}, n \in \mathbb{N}$ be a sequence of nonnegative Borel functions satisfying

$$
\sup _{n \in \mathbb{N}, x \in E} g_{n}(x)<+\infty
$$

and assume that

1. as $n \rightarrow \infty$ the sequence $g_{n}$ converges, $\mu$-almost surely, to a Borel function $g$;

2. the operator $1+(g-1) \Pi$ is invertible;

3. $\left\|\left(g_{n}-g\right) \Pi\right\| \rightarrow 0$.

Then

$$
\Pi^{g_{n}} \rightarrow \Pi^{g} \quad \text { in } \mathscr{I}_{1, \mathrm{loc}}(E, \mu)
$$

PROOF. Let $B$ be a bounded set and write

$$
\chi_{B} \Pi^{g} \chi_{B}=\left(\sqrt{g} \chi_{B} \Pi\right)\left(\Pi(1+(g-1) \Pi)^{-1} \Pi\right)\left(\Pi \chi_{B} \sqrt{g}\right) .
$$

If $A$ ranges in the space of Hilbert-Schmidt operators and $D$ in the space of bounded self-adjoint operators endowed with the operator norm, then the correspondence $(A, D) \rightarrow A^{*} D A$ induces a continuous mapping into the space of 
trace-class operators. By our third assumption, the operators $1+\left(g_{n}-1\right) \Pi$ converge to $1+(g-1) \Pi$ in the norm topology, consequently, for large $n$ the operators $1+\left(g_{n}-1\right) \Pi$ are invertible, the inverses also converge in norm, and, finally, $\Pi\left(1+\left(g_{n}-1\right) \Pi\right)^{-1} \Pi \rightarrow \Pi(1+(g-1) \Pi)^{-1} \Pi$ in the norm topology. Uniform boundedness (38) of $g_{n}$ and pointwise convergence of $g_{n}$ imply that $\sqrt{g}_{n} \chi_{B} \Pi \rightarrow \sqrt{g} \chi_{B} \Pi$ in the Hilbert-Schmidt norm, and the proof is complete.

2.12. Normalized multiplicative functionals. If the multiplicative functional $\Psi_{g}$ is $\mathbb{P}_{\Pi \text {-integrable, then we introduce the normalized multiplicative functional }}$ $\bar{\Psi}_{g}$ by the formula

$$
\bar{\Psi}_{g}=\frac{\Psi_{g}}{\int_{\operatorname{Conf}(E)} \Psi_{g} d \mathbb{P}_{\Pi}} .
$$

We reformulate Proposition 2.1 in [7] in our new notation (37), (39).

Proposition 2.7. If $g$ is a bounded Borel function on E such that

$$
\sqrt{g-1} \Pi \sqrt{g-1} \in \mathscr{I}_{1}(E, \mu)
$$

and the operator $1+(g-1) \Pi$ is invertible, then the subspace $\sqrt{g} L$ is closed, the normalized multiplicative functional $\bar{\Psi}_{g}$ is well defined, and we have

$$
\bar{\Psi}_{g} \mathbb{P}_{\Pi}=\mathbb{P}_{\Pi g} \text {. }
$$

Note that closedness of the subspace $\sqrt{g} L$ is immediate from the invertibility of the operator $1+(g-1) \Pi$ : indeed, the operator $1+(g-1) \Pi$ takes the subspace $L$ to the subspace $g L$, which is consequently closed. Since the function $g$ is bounded from above, the subspace $\sqrt{g} L$ is, a fortiori, closed as well: indeed, if $\psi_{n} \in L$ are such that $\sqrt{g} \psi_{n} \rightarrow \psi$, then, by boundedness of $g$, we also have $g \psi_{n} \rightarrow \sqrt{g} \psi$, so, by closedness of the space $g L$, we have $\sqrt{g} \psi \in g L$ whence also $\psi \in \sqrt{g} L$ [this implication is valid even if the set $\{x: g(x)=0\}$ has positive measure since, by definition, $\left.\psi=\chi_{\{x: g(x)>0\}} g\right]$.

A key point in the argument of this paper is that the normalized multiplicative functional (39) can be defined, in such a way that the formula (40) still holds, even when the multiplicative functional $\Psi_{g}$ itself is not defined, see Proposition 4.2 below.

2.13. On the subspace $\sqrt{g} L$. We now give another sufficient condition for closedness of the subspace $\sqrt{g} L$ for a bounded function $g$. If the function $g$ is bounded away from zero in addition to being bounded from above, then the subspace $\sqrt{g} L$ is automatically closed. If $\inf _{x \in E} g=0$, then we prepare the following simple proposition. Recall that $\|A\|$ always stands for the usual operator norm of a bounded operator $A$. 
PROPOSITION 2.8. Let $\Pi$ be an operator of orthogonal projection onto a closed subspace $L \subset L_{2}(E, \mu)$, and let $C \subset E$ be a Borel subset such that $\left\|\chi_{E \backslash C} \Pi\right\|<1$. Then the subspace $\chi_{C} L$ is closed and the natural restriction map $\varphi \rightarrow \chi_{C} \varphi$ induces an isomorphism of Hilbert spaces $L$ and $\chi_{C} L$.

PROOF. Indeed, our assumptions imply the existence of a positive constant $\alpha$ such that for any $\varphi \in L$ we have

$$
\left\|\chi_{E \backslash C} \varphi\right\| \leq \alpha\left\|\chi_{C} \varphi\right\|,
$$

and the proposition follows.

REMARK. In the proposition above, we have not used the local trace class assumption. In the case in which $\Pi$ is locally trace class and $E \backslash C$ is bounded, it suffices to check that the range of $\Pi$ does not admit functions supported in $E \backslash C$, whence it follows that the compact operator $\chi_{E \backslash C} \Pi$ is a strict contraction.

COROLlaRY 2.9. Let $g$ be a bounded nonnegative Borel function on $E$ and $C \subset E$ a Borel subset such that

1. $\left\|\chi_{E \backslash C} \Pi\right\|<1$;

2. the function $g$ in restriction to $C$ is bounded away from 0 .

Then the subspace $\sqrt{g} L$ is closed.

Proof. If $\left.g\right|_{C}>\varepsilon>0$ and $g<M<+\infty$ on the whole space $E$, then the inequality (41) implies, for any $\psi \in \sqrt{g} L$, the estimate

$$
\left\|\chi_{E \backslash C} \psi\right\| \leq M \varepsilon^{-1} \alpha\left\|\chi_{C} \psi\right\|
$$

The subspace $\chi_{C} L$ is closed by our second assumption, and so is $\chi_{C} \sqrt{g} L$. By (42), for all $\psi \in \sqrt{g} L$, the natural restriction map $\psi \rightarrow \chi_{C} \psi$ is invertible with bounded inverse, and the proposition follows.

Proposition 2.10. Let $g: E \rightarrow[0,1]$ be a Borel function such that

1. $\operatorname{tr}\left(\chi_{\{x \in E: g(x)<1\}} \Pi \chi_{\{x \in E: g(x)<1\}}\right)<+\infty$,

2. a function $\varphi \in L$ satisfying $\chi_{\{x: g(x)<1\}} \varphi=\varphi$ must be the zero function.

Then all the conclusions of Proposition 2.7 hold for the function $g$.

ProOF. Our assumptions imply $\left\|\chi_{\{x \in E: g(x)<1\}} \Pi\right\|<1$, whence the operator $1+(g-1) \Pi$ is invertible. The space $\sqrt{g} L$ is closed by Corollary 2.9. 
2.14. On invertibility of the operator $1+(g-1) \Pi$. Take a nonnegative bounded Borel function $g$ such that the operator $\sqrt{g-1} \Pi$ is Hilbert-Schmidt. It follows that the operator $(g-1) \Pi$ is also Hilbert-Schmidt, while the operator $\sqrt{g-1} \Pi \sqrt{g-1}$ is trace-class. In particular, since all these operators are compact, invertibility of the operator $1+(g-1) \Pi$ is equivalent to its injectivity, and the same is true for $1+\sqrt{g-1} \Pi \sqrt{g-1}$. We start with a simple remark.

PROPOSITION 2.11. 1. The invertibility of the operator $1+(g-1) \Pi$ is equivalent to the invertibility of the operator $1+\sqrt{g-1} \Pi \sqrt{g-1}$.

2. If $\sup |g-1|<1$, then the operator $1+(g-1) \Pi$ is invertible.

PROOF. Indeed, if $\sqrt{g-1} \Pi \sqrt{g-1} \varphi=-\varphi$, then $(g-1) \Pi(\sqrt{g-1} \varphi)=$ $-\sqrt{g-1} \varphi$. Conversely, if $(g-1) \Pi \varphi=-\varphi$, then the function $\psi=\sqrt{g-1} \Pi \varphi$ satisfies $\sqrt{g-1} \psi=-\varphi$ and, consequently, $\sqrt{g-1} \Pi \sqrt{g-1} \psi=-\psi$, and the first item is proved. The second item is clear since if $\sup |g-1|<1$, then $\|(g-1) \Pi\|<1$.

We next show that perturbing a positive function $g$ on a bounded set does not change the invertibility of the operator $1+(g-1) \Pi$.

PROPOSITION 2.12. Let $g_{1}, g_{2}$ be positive bounded Borel functions such that the operators $\sqrt{g_{1}-1} \Pi, \sqrt{g_{2}-1} \Pi$ are Hilbert-Schmidt and the set $\left\{x: g_{1}(x) \neq\right.$ $\left.g_{2}(x)\right\}$ is bounded. Then the invertibility of the operator $1+\left(g_{1}-1\right) \Pi$ is equivalent to the invertibility of the operator $1+\left(g_{2}-1\right) \Pi$.

PROOF. We have $\mathbb{E}_{\mathbb{P}_{\Pi}} \Psi_{g_{i}}=\operatorname{det}\left(1+\sqrt{g_{i}-1} \Pi \sqrt{g_{i}-1}\right), i=1$, 2. Since our functions are positive, the operator $1+\left(g_{i}-1\right) \Pi$ is invertible if and only if the multiplicative functional $\Psi_{g_{i}}$ is positive on a subset of positive $\mathbb{P}_{\Pi \text {-measure of }}$ $\operatorname{Conf}(E)$, or, in other words, if and only if the infinite product $\prod_{x \in X} g_{i}(x)$ converges absolutely to a positive limit with positive $\mathbb{P}_{\Pi}$-probability. Since both functions are positive and the set $\left\{x: g_{1}(x) \neq g_{2}(x)\right\}$ is bounded, for $\mathbb{P}_{\Pi \text {-almost any }}$ configuration $X$ the functions $g_{1}$ and $g_{2}$ are equal except on finitely many particles of $X$, and the infinite products $\prod_{x \in X} g_{i}(x), i=1,2$, either both converge or both diverge.

PROPOSITION 2.13. If $g$ is a nonnegative bounded Borel function satisfying

1. the operator $(g-1) \Pi$ is compact;

2. the operator $1+(g-1) \Pi$ is invertible,

then, for any subset $C \subset E$, the function $g^{C}=\chi_{E \backslash C}+g \chi_{C}$ also satisfies the same conditions (1), (2). 
Proof. Compactness of $\left(g^{C}-1\right) \Pi=\chi_{C}(g-1) \Pi$ is clear. If $1+\left(g^{C}-1\right) \Pi$ fails to be invertible and has a nontrivial kernel, then there exists a function $\psi$ satisfying $\psi+\chi_{C}(g-1) \Pi \psi=0$. It follows that $\psi=\chi_{C} \psi$ and that $\psi+(g-$ 1) $\Pi \psi=0$, a contradiction that completes the proof.

2.15. Inverting self-adjoint operators. It is sometimes more convenient to take the inverse of $1+\sqrt{g-1} \Pi \sqrt{g-1}$ rather than that of $1+(g-1) \Pi$, and we rewrite the formula (37) in the following way. Let $\alpha>0$, let $g$ be a Borel function, perhaps unbounded from above, on $E$, satisfying $g>1+\alpha$ and such that the operator $\sqrt{g-1} \Pi$ is Hilbert-Schmidt. Then $\sqrt{g} L$ is a subspace of $L_{2}(E, \mu)$, automatically a closed one; we let $\Pi^{g}$ be the operator of orthogonal projection onto $\sqrt{g} L$ and set $\Pi_{g}=\sqrt{g-1} \Pi \sqrt{g-1}$. The operator $1+\Pi_{g}$ is then automatically invertible.

\section{Proposition 2.14. We have}

$$
\Pi^{g}=\sqrt{1+(g-1)^{-1}} \Pi_{g}\left(1+\Pi_{g}\right)^{-1} \sqrt{1+(g-1)^{-1}} .
$$

PROOF. If $g$ is bounded, then, noting that invertibility of $1+\Pi_{g}$ implies that of $1+(g-1) \Pi$, we again use the identity $p(1+q p)^{-1}=(1+p q)^{-1} p$, valid for arbitrary bounded operators $p, q$ once both sides are well defined. If $g$ is unbounded, then we approximate $g$ from below by a sequence of bounded functions and pass to the limit in the space of Hilbert-Schmidt operators.

REMARK. While this proposition is sufficient for our purposes, the equality (43) still holds under much milder assumptions on $g$; the key point is to ensure invertiblity of the operators $1+\Pi_{g}, 1+(g-1) \Pi$. The formula (43) implies the following analogue of Proposition 2.6.

Proposition 2.15. Let $\alpha>0$, and let $g$ be a Borel fucntion on E satisfying $g>1+\alpha, \sqrt{g-1} \Pi \in \mathscr{I}_{2}(E, \mu)$. Let $g_{n}, n \in \mathbb{N}$ be a sequence of nonnegative bounded Borel functions satisfying $1+\alpha \leq \inf _{n \in \mathbb{N}, x \in E} g_{n}(x)$, and assume that $\sqrt{g_{n}-1} \Pi \rightarrow \sqrt{g-1} \Pi \in \mathscr{I}_{2}(E, \mu)$. Then

$$
\Pi^{g_{n}} \rightarrow \Pi^{g} \quad \text { in } \mathscr{I}_{1, \operatorname{loc}}(E, \mu) .
$$

PROOF. The Hilbert-Schmidt convergence of the sequence $\sqrt{g_{n}-1} \Pi \mathrm{im}$ plies the trace class, and, a fortiori, norm, convergence of the sequence $\Pi_{g_{n}}$. The proof is now concluded in the same way as that of Proposition 2.6.

2.16. Palm measures of determinantal point processes. Palm measures of determinantal point processes admit the following characterization. As above, let $\Pi \in \mathscr{I}_{1, \operatorname{loc}}(E, \mu)$ be the operator of orthogonal projection onto a closed subspace 
$L \subset L_{2}(E, \mu)$. For $q \in E$ satisfying $\Pi(q, q) \neq 0$, introduce a kernel $\Pi^{q}$ by the formula

$$
\Pi^{q}(x, y)=\Pi(x, y)-\frac{\Pi(x, q) \Pi(q, y)}{\Pi(q, q)} .
$$

If $\Pi(q, q)=0$, then we also have $\Pi(x, q)=\Pi(q, y)=0$ almost surely with respect to $\mu$, and we set $\Pi^{q}=\Pi$.

The operator $\Pi^{q}$ defines an orthogonal projection onto the subspace

$$
L(q)=\{\varphi \in L: \varphi(q)=0\}
$$

of functions in $L$ that assume the value zero at the point $q$; the space $L(q)$ is well defined by Assumption 1; in other words, $L(q)$ is the orthogonal complement of $v_{q}$ in $L$. Iterating, let $q_{1}, \ldots, q_{l} \in E$ be distinct and set

$$
L\left(q_{1}, \ldots, q_{l}\right)=\left\{\varphi \in L: \varphi\left(q_{1}\right)=\cdots=\varphi\left(q_{l}\right)=0\right\},
$$

and let $\Pi^{q_{1}, \ldots, q_{l}}$ be the operator of orthogonal projection onto the subspace $L\left(q_{1}, \ldots, q_{l}\right)$. Shirai and Takahashi [26] have proved:

Proposition 2.16 (Shirai and Takahashi [26]). For any $l \in \mathbb{N}$ and for $\rho_{l^{-}}$ almost every $l$-tuple $q_{1}, \ldots, q_{l}$ of distinct points in $E$, the iterated reduced Palm measure $\mathbb{P}_{\Pi}^{q_{1}, \ldots, q_{l}}$ is given by the formula

$$
\mathbb{P}_{\Pi}^{q_{1}, \ldots, q_{l}}=\mathbb{P}_{\Pi^{q_{1}, \ldots, q_{l}}} .
$$

REMARK. Shirai and Takahashi [26] have in fact established the formula (45) for arbitrary positive self-adjoint locally trace-class contractions; the formula (44) for the kernel stays the same. Note that in the discrete case the formula for contractions is a corollary of the formula for projection operators, since formula (45) is local (both in the discrete and the continuous cases) and contractions are reduced to projections by doubling the phase space (see Lyons [19]).

2.17. Conditional measures in the discrete case. In this subsection, we consider the discrete case, in which the space $E$ is a countable set endowed with the discrete topology, and the measure $\mu$ is the counting measure. In this case, the reduced Palm measure $\mathbb{P}^{q}$ of a point process $\mathbb{P}$ on $\operatorname{Conf}(E)$ can be described as follows: one takes the conditional measure of $\mathbb{P}$ on the subset of configurations containing a particle at position $q$, and then one removes the particle at $q$; more formally, $\mathbb{P}^{q}$ is the push-forward of the said conditional measure under the operation that to a configuration $X$ containing the particle at $q$ assigns the configuration $X \backslash\{q\}$.

In the discrete case, we also have a dual construction: let $\mathbb{P}^{\breve{q}}$ be the conditional measure of $\mathbb{P}$ with respect to the event that there is no particle at position $q$. More formally, set

$$
\operatorname{Conf}(E ; E \backslash\{q\})=\{X \in \operatorname{Conf}(E): q \notin X\},
$$


and write

$$
\mathbb{P}^{\breve{q}}=\frac{\left.\mathbb{P}\right|_{\operatorname{Conf}(E ; E \backslash\{q\})}}{\mathbb{P}(\operatorname{Conf}(E ; E \backslash\{q\}))}
$$

be the normalized restriction of $\mathbb{P}$ onto the subset $\operatorname{Conf}(E ; E \backslash\{q\})$.

We have a dual to Proposition 2.16.

Proposition 2.17. Let $q \in E$ be such that $\mu(\{q\})>0$. Then the operator of orthogonal projection onto the subspace $\chi_{E \backslash q} L$ has the kernel $\Pi^{\breve{q}}$ given by the formula

$$
\begin{aligned}
& \Pi^{\breve{q}}(x, y)=\Pi(x, y)+\frac{\Pi(x, q) \Pi(q, y)}{1-\Pi(q, q)}, \quad x \neq q, y \neq q ; \\
& \Pi^{\breve{q}}(x, q)=\Pi^{\breve{q}}(q, y)=0, \quad x, y \in E .
\end{aligned}
$$

PROOF. This is a particular case of Corollary 6.4 in Lyons [19]; see also Shirai-Takahashi [26, 27] and Borodin-Rains [4].

Given $l \in \mathbb{N}, m<l$ and an $l$-tuple $\left(p_{1}, \ldots, p_{l}\right)$, of distinct points in $E$, recall that we have introduced a subspace $L\left(p_{1}, \ldots, p_{m}, \breve{p}_{m+1}, \ldots, \breve{p}_{l}\right)$ by the formula

$$
\begin{aligned}
& L\left(p_{1}, \ldots, p_{m}, \breve{p}_{m+1}, \ldots, \breve{p}_{l}\right) \\
& \quad=\left\{\chi_{E \backslash\left\{p_{m+1}, \ldots, p_{l}\right\}} \varphi: \varphi \in L, \varphi\left(p_{1}\right)=\cdots=\varphi\left(p_{m}\right)=0\right\} .
\end{aligned}
$$

Let $\Pi^{p_{1}, \ldots, p_{m}, \breve{p}_{m+1}, \ldots, \breve{p}_{l}}$ be the operator of orthogonal projection onto the subspace $L\left(p_{1}, \ldots, p_{m}, \breve{p}_{m+1}, \ldots, \breve{p}_{l}\right)$. The corresponding determinantal measure

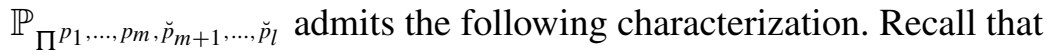

$$
\mathbf{C}\left(p_{1}, \ldots, p_{m}, \breve{p}_{m+1}, \ldots, \breve{p}_{l}\right)
$$

is the set of configurations on $E$ containing exactly one particle in each of the positions $p_{1}, \ldots, p_{m}$ and no particles in the positions $p_{m+1}, \ldots, p_{l}$. There is a natural erasing bijection between $\mathbf{C}\left(p_{1}, \ldots, p_{m}, \breve{p}_{m+1}, \ldots, \breve{p}_{l}\right)$ and $\mathbf{C}\left(\breve{p}_{1}, \ldots, \breve{p}_{m}\right.$, $\breve{p}_{m+1}, \ldots, \breve{p}_{l}$ ) obtained by erasing the particles in positions $p_{1}, \ldots, p_{m}$.

PROPOSITION 2.18. Consider the normalized restriction of $\mathbb{P}_{\Pi}$ onto the set $\mathbf{C}\left(p_{1}, \ldots, p_{m}, \breve{p}_{m+1}, \ldots, \breve{p}_{l}\right)$. The push-forward of this normalized restriction onto the set $\mathbf{C}\left(\breve{p}_{1}, \ldots, \breve{p}_{m}, \breve{p}_{m+1}, \ldots, \breve{p}_{l}\right)$ under the erasing bijection is the mea-

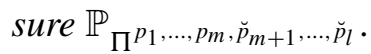

ProOF. Again, this is a reformulation of Corollary 6.4 in Lyons [19]; see also Shirai-Takahashi $[26,27]$. 
2.18. Action of Borel automorphisms on point processes. Let $T: E \rightarrow E$ be an invertible measurable map such that for any bounded set $B \subset E$ the set $T(B)$ is also bounded. The map $T$ naturally acts on the space of configurations $\operatorname{Conf}(E)$ : namely, given $X \in \operatorname{Conf}(E)$ we set

$$
T(X)=\{T x, x \in X\} .
$$

Note that, by our assumptions, $T(X)$ is a well-defined configuration on $E$; slightly abusing notation, we thus keep the same symbol $T$ for the induced action on the space of configurations.

Let $\mathbb{P}$ be a probability measure on $\operatorname{Conf}(E)$. We assume that $\mathbb{P}$ admits correlation measures of all orders, and, for $l \in \mathbb{N}$, we let $\rho_{l}$ be the $l$ th correlation measure of the point process $\mathbb{P}$. The $l$ th Cartesian power of $T$ naturally acts on the measure $\rho_{l}$, and, slightly abusing notation, we denote the resulting measure by $\rho_{l} \circ T$. The measure $\rho_{l} \circ T$ is, of course, the $l$ th correlation measure of the point process $\mathbb{P} \circ T$, the push-forward of the measure $\mathbb{P}$ under the induced action of the automorphism $T$ on the space of configurations.

We now prove a simple general statement: if for a point process $\mathbb{P}$ and an arbitrary fixed $l \in \mathbb{N}$, the reduced Palm measures corresponding to different $l$-tuples of points are equivalent, then for any Borel automorphism $T$ acting by the identity beyond a bounded set, the measures $\mathbb{P}$ and $\mathbb{P} \circ T$ are also equivalent, and the Radon-Nikodym derivative is found explicitly in terms of the Radon-Nikodym derivatives of the reduced Palm measures. More precisely, we have the following proposition.

Proposition 2.19. Let $T: E \rightarrow E$ be a Borel automorphism admitting a bounded subset $B \subset E$ such that $T(x)=x$ for all $x \in E \backslash B$. Assume that

1. for any $l \in \mathbb{N}$, the correlation measures $\rho_{l}$ and $\rho_{l} \circ T$ are equivalent;

2. for any two collections $\left\{q_{1}, \ldots, q_{l}\right\}$ and $\left\{q_{1}^{\prime}, \ldots, q_{l}^{\prime}\right\}$ of distinct points of $E$, the measures $\mathbb{P}^{q_{1}, \ldots, q_{l}}$ and $\mathbb{P}_{1}^{q_{1}^{\prime}, \ldots, q_{l}^{\prime}}$ are equivalent.

Then the measures $\mathbb{P}$ and $\mathbb{P} \circ T$ on $\operatorname{Conf}(E)$ are equivalent, and for $\mathbb{P}$-almost every configuration $X \in \operatorname{Conf}(E)$ such that $X \cap B=\left\{q_{1}, \ldots, q_{l}\right\}$ we have

$$
\frac{d \mathbb{P} \circ T}{d \mathbb{P}}(X)=\frac{d \mathbb{P}^{T q_{1}, \ldots, T q_{l}}}{d \mathbb{P}^{q_{1}, \ldots, q_{l}}}\left(X \backslash\left\{q_{1}, \ldots, q_{l}\right\}\right) \times \frac{d \rho_{l} \circ T}{d \rho_{l}}\left(q_{1}, \ldots, q_{l}\right) .
$$

Proof. Let $l \in \mathbb{N}$, let $\mathbb{P}, \widetilde{\mathbb{P}}$ be probability measures on $\operatorname{Conf}(E)$ admitting correlation measures of order $l$, denoted, respectively, $\rho_{l}, \widetilde{\rho}_{l}$. Let $\mathbb{P}^{q_{1}}, \ldots, q_{l}, \widetilde{\mathbb{P}}^{q_{1}}, \ldots, q_{l}$ stand for the respective reduced Palm measures. The symbol $\ll$ denotes absolute continuity of measures. Proposition 2.19 follows from:

PROPOSITION 2.20. If $\widetilde{\rho}_{l} \ll \rho_{l}$ and $\widetilde{\mathbb{P}}^{q_{1}, \ldots, q_{l}} \ll \mathbb{P}^{q_{1}, \ldots, q_{l}}$ for $\rho_{l}$-almost any distinct $q_{1}, \ldots, q_{l} \in E$, then also $\widetilde{\mathbb{P}} \ll \mathbb{P}$ and for $\mathbb{P}$-almost any $X \in \operatorname{Conf}(E)$ and any 
l particles $q_{1}, \ldots, q_{l} \in X$ we have

$$
\frac{d \widetilde{\mathbb{P}}}{d \mathbb{P}}=\frac{d \widetilde{\mathbb{P}}^{q_{1}, \ldots, q_{l}}}{d \mathbb{P} q_{1}, \ldots, q_{l}}\left(X \backslash\left\{q_{1}, \ldots, q_{l}\right\}\right) \times \frac{d \widetilde{\rho}_{l}}{d \rho_{l}}\left(q_{1}, \ldots, q_{l}\right) .
$$

Proof. We use Proposition 2.1. Take $X_{0} \in \operatorname{Conf}(E)$ and $q_{1}^{(0)}, \ldots, q_{l}^{(0)} \in X_{0}$; take disjoint bounded open sets $B^{(1)}, \ldots, B^{(l)} \subset E$, set $B=\bigcup B^{(i)}$ and take an open set $U \subset E$ disjoint from all $B^{(i)}$ in such a way that $q_{i}^{(0)} \in B^{(i)}$ for all $i=1, \ldots, l$ and $X_{0} \backslash\left\{q_{1}^{(0)}, \ldots, q_{l}^{(0)}\right\} \subset U$. Let $\mathscr{W}$ be a neighbourhood of $X_{0} \backslash\left\{q_{1}^{(0)}, \ldots, q_{l}^{(0)}\right\}$ in $\operatorname{Conf}(E)$ satisfying $\mathscr{W} \subset\{X \in \operatorname{Conf}(E): X \subset U\}$. Introduce a neighbourhood $\mathscr{Z}$ of $X_{0}$ by setting

$$
\mathscr{Z}=\left\{X \in \operatorname{Conf}(E): \#_{B^{(1)}}(X)=\cdots=\#_{B^{(l)}}(X)=1,\left.X\right|_{E \backslash B} \subset \mathscr{W}\right\} .
$$

Sets given by (49) form a basis of neighbourhoods of $X_{0}$. We prepare a simple general lemma.

LEMMA 2.21. Let $v_{1}$ and $v_{2}$ be two Borel probability measures on a complete separable metric space $Y$. Let $\mathscr{V}=\{V\}$ be a basis of neighbourhoods on $Y$. Let $\varphi$ be a nonnegative function on $Y$ such that for any neighbourhood $V \in \mathscr{V}$ we have

$$
v_{1}(V)=\int_{V} \varphi d v_{2}
$$

Then $\nu_{1} \ll v_{2}$ and $d v_{1} / d \nu_{2}=\varphi$ almost surely with respect to $\nu_{2}$.

PROOF. Indeed, if the equality (50) holds for a basis of neighbourhoods, then, due to tightness, it holds for all open sets and then also for all Borel sets.

By definition of Palm measures and Proposition 2.1, we have

$$
\mathbb{P}(\mathscr{Z})=\int_{B^{(1)} \times \cdots \times B^{(l)}} \mathbb{P}^{q_{1}, \ldots, q_{l}}(\mathscr{W}) d \rho_{l}\left(q_{1}, \ldots, q_{l}\right) .
$$

A similar formula holds for $\widetilde{\mathbb{P}}$. Taking $X \in \mathscr{Z}$, setting $q_{i}=X \cap B^{(i)}$, and using the assumption $\widetilde{\mathbb{P}} q_{1}, \ldots, q_{l} \ll \mathbb{P}^{q_{1}, \ldots, q_{l}}$, we therefore have

$$
\widetilde{\mathbb{P}}(\mathscr{Z})=\int_{\mathscr{Z}} \frac{d \widetilde{\mathbb{P}}^{q_{1}, \ldots, q_{l}}}{d \mathbb{P}^{q_{1}, \ldots, q_{l}}}\left(X \backslash\left\{q_{1}, \ldots, q_{l}\right\}\right) \times \frac{d \widetilde{\rho}_{l}}{d \rho_{l}}\left(q_{1}, \ldots, q_{l}\right) d \mathbb{P}(X) .
$$

Since the formula holds for an arbitrary neighbourhood of the form (49), Proposition 2.20 follows from Lemma 2.21.

We now derive Proposition 2.19 from Proposition 2.20. As before, let $\operatorname{Conf}(E ; E \backslash B)$ be the subset of those configurations on $E$ all whose particles 
lie in $E \backslash B$. Since the automorphism $T$ acts by the identity on $E \backslash B$, all configurations in the set $\operatorname{Conf}(E ; E \backslash B)$ are fixed by $T$, and we have

$$
\left.\mathbb{P}^{q_{1}, \ldots, q_{l}}\right|_{\operatorname{Conf}(E ; E \backslash B)} \circ T=\left.\mathbb{P}^{T q_{1}, \ldots, T q_{l}}\right|_{\operatorname{Conf}(E ; E \backslash B)} .
$$

By definition, we have $X \backslash\left\{q_{1}, \ldots, q_{l}\right\} \in \operatorname{Conf}(E ; E \backslash B)$, whence, again using neighbourhoods of the form (49) as well as Lemma 2.21, we obtain

$$
\frac{d \mathbb{P}^{q_{1}, \ldots, q_{l}} \circ T}{d \mathbb{P}^{q_{1}, \ldots, q_{l}}}\left(X \backslash\left\{q_{1}, \ldots, q_{l}\right\}\right)=\frac{d \mathbb{P}^{T q_{1}, \ldots, T q_{l}}}{d \mathbb{P}^{q_{1}, \ldots, q_{l}}}\left(X \backslash\left\{q_{1}, \ldots, q_{l}\right\}\right),
$$

and Proposition 2.19 follows now from Proposition 2.20.

\section{The relation between Palm subspaces.}

3.1. The case of continuous measures. Proposition 2.19 shows that in order to establish the quasi-invariance under the group of compactly supported diffeomorphisms for a point process, it suffices to show that its reduced Palm measures of the same order are equivalent. In this section, we show that Assumption 2 implies the relation (51) between Palm subspaces; we then show that integrable kernels satisfy Assumption 2. The transition from relation (51) to the equivalence of Palm measures is achieved in the next section [cf. Corollary 4.11 and the formula (90)].

As before, we consider a closed subspace $L \subset L_{2}(E, \mu)$ such that the corresponding orthogonal projection $\Pi$ is locally trace class with a kernel satisfying Assumptions 1,2 . Assume that the measure $\mu$ satisfies $\mu(\{p\})=0$ for any $p \in \mathbb{R}$.

Proposition 3.1. For any distinct points $p_{1}, \ldots, p_{l}, q_{1}, \ldots, q_{l} \in U$ we have

$$
L\left(p_{1}, \ldots, p_{l}\right)=\frac{\left(x-p_{1}\right) \cdots\left(x-p_{l}\right)}{\left(x-q_{1}\right) \cdots\left(x-q_{l}\right)} L\left(q_{1}, \ldots, q_{l}\right) .
$$

REMARK. The coincidence of subspaces is understood as coincidence of subspaces in $L_{2}$; the functions from the right-hand side subspace are of course not defined at the points $q_{1}, \ldots, q_{l}$; they are nonetheless well defined as elements of $L_{2}$ since the measure $\mu$ is continuous. For discrete measures the formulation will be modified.

Proof. In the continuous case, Assumption 2 implies, for any $q \in U$, the inclusion $\frac{L(q)}{x-q} \subset L$, whence $\frac{x-p_{1}}{x-q_{1}} L\left(q_{1}\right) \subset L\left(q_{1}\right)+\frac{L\left(q_{1}\right)}{x-q_{1}} \subset L$, Any function $\varphi \in$ $\frac{x-p_{1}}{x-q_{1}} L\left(q_{1}\right)$ satisfies $\varphi\left(p_{1}\right)=0$, whence $\frac{x-p_{1}}{x-q_{1}} L\left(q_{1}\right) \subset L\left(p_{1}\right)$. Interchanging the points $p_{1}$ and $q_{1}$, we obtain the converse inclusion (using again continuity of the measure $\mu$ ), and the proposition is proved for $l=1$. Proposition 1.1 implies that if $\Pi$ satisfies Assumption 2, then so does $\Pi^{p}$ for any $p \in U$, and the proof is completed by induction on $l$. 
3.2. The case of discrete measures. We now let $E$ be a countable discrete subset of $\mathbb{R}$ endowed with the counting measure $\mu$.

Proposition 3.2. Let the kernel $\Pi$ satisfy Assumption 2. Let $p_{1}, \ldots, p_{l} \in E$ be distinct, and let $\pi$ be a permutation of $\{1, \ldots, l\}$. Then we have

$$
\begin{aligned}
& L\left(p_{\pi(1)}, \ldots, p_{\pi(m)}, \breve{p}_{\pi(m+1)}, \ldots, \breve{p}_{\pi(l)}\right) \\
& =\chi_{E \backslash\left\{p_{1}, \ldots, p_{l}\right\}}(x) \frac{\left(x-p_{\pi(1)}\right) \cdots\left(x-p_{\pi(m)}\right)}{\left(x-p_{1}\right) \cdots\left(x-p_{m}\right)} \\
& \quad \times L\left(p_{1}, \ldots, p_{m}, \breve{p}_{m+1}, \ldots, \breve{p}_{l}\right) .
\end{aligned}
$$

ProOF. As in the continuous case, we proceed by induction and start with the case $l=2, m=1$ : we need to show, for any distinct $p, q \in E$, the equality

$$
L(p, \breve{q})=\chi_{E \backslash\{p, q\}} \frac{x-p}{x-q} L(q, \breve{p}) .
$$

Now, by Proposition 3.4, we have

$$
\chi_{E \backslash q} \frac{L(q)}{x-q} \subset \chi_{E \backslash q} L .
$$

Since

$$
\frac{x-p}{x-q}=1+\frac{q-p}{x-q}
$$

we also have

$$
\frac{x-p}{x-q} \chi_{E \backslash q} L(q) \subset \chi_{E \backslash q} L=L(\breve{q}) .
$$

Now, multiplying any function by $\chi_{E \backslash q} \frac{x-p}{x-q}$ yields a function that assumes value 0 at the point $p$; we thus conclude

$$
\chi_{E \backslash\{p, q\}} \frac{x-p}{x-q} L(\breve{p}, q) \subset L(p, \breve{q}) .
$$

Interchanging the variables $p, q$, we obtain the inverse inclusion, and (53) is proved. If $\Pi$ satisfies Assumption 2, then, by Proposition 1.1, so does $\Pi^{p}$, and, by definition, so does $\Pi^{\breve{p}}$. Induction completes the proof. 


\subsection{Proof of Proposition 1.2.}

\subsubsection{The subspace $L^{\prime}$.}

Proposition 3.3. If $\varphi \in L_{2}(\mathbb{R}, \mu)$ is such that $x \varphi \in L_{2}(\mathbb{R}, \mu)$, then the integrals

$$
\int_{\mathbb{R}} \varphi(x) A(x) d \mu(x), \quad \int_{\mathbb{R}} \varphi(x) B(x) d \mu(x)
$$

are well defined.

Proof. Since for any $p \in U$ we have

$$
\left\langle v_{p}(x),(x-p) \varphi(x)\right\rangle=A(p) \int_{\mathbb{R}} \varphi(x) B(x) d \mu(x)-B(p) \int_{\mathbb{R}} \varphi(x) A(x) d \mu(x),
$$

keeping in mind that linear independence of $A$ and $B$ implies the existence of points $p_{1}, p_{2} \in U$ such that $A\left(p_{1}\right) B\left(p_{2}\right)-A\left(p_{2}\right) B\left(p_{1}\right) \neq 0$, we obtain the desired integrability.

Let

$$
\begin{aligned}
L^{\prime}= & \left\{\psi \in L: x \psi \in L_{2}(\mathbb{R}, \mu),\right. \\
& \left.\int_{\mathbb{R}} \psi(x) A(x) d \mu(x)=\int_{\mathbb{R}} \psi(x) B(x) d \mu(x)=0\right\} .
\end{aligned}
$$

Proposition 3.4. Let $p \in U$ and $\varphi \in L$ satisfy $\varphi(p)=0$. Then there exists $\psi \in L^{\prime}$ such that

$$
\varphi(x)=(x-p) \psi(x) .
$$

ProOF. It suffices to consider the case $p=0, A(0)=0, B(0) \neq 0$ : since the functions $A, B$ are linearly independent, the general case is reduced to this particular one by a translation of $\mathbb{R}$ and a linear unimodular change of variable (8). Let $\psi^{\prime}$ be such that (56) holds [in the continuous case, such a function $\psi^{\prime}$ is unique: we simply set $\psi^{\prime}=\varphi(x) /(x-p)$ and note that $\psi^{\prime}$ is square-integrable due to Assumption 1 ; in the discrete case, however, there are many such functions, differing by their value at $p=0]$. Using the integrable form of our kernel and the relation $\varphi \in L$, write

$$
\begin{aligned}
(x \Pi-\Pi x) \psi^{\prime} & =x \Pi \psi^{\prime}(x)-\varphi(x) \\
& =A(x) \int_{\mathbb{R}} B(y) \psi^{\prime}(y) d \mu(y)-B(x) \int_{\mathbb{R}} A(y) \psi^{\prime}(y) d \mu(y) .
\end{aligned}
$$


Since $\varphi \in L, \varphi(0)=0, A(0)=0, B(0) \neq 0$, substituting $x=0$ into (57) we obtain

$$
\int_{\mathbb{R}} A(y) \psi^{\prime}(y) d \mu(y)=0 .
$$

Recall that by definition we have

$$
v_{0}(x)=\frac{A(x)}{x} \in L .
$$

Dividing (57) by $x$ and keeping (58) in mind, we obtain that there exists $\alpha \in \mathbb{C}$ such that

$$
\Pi \psi^{\prime}(x)-\psi(x)-\alpha \delta_{0}=v_{0}(x) \int_{\mathbb{R}} B(y) \psi^{\prime}(y) d \mu(y) .
$$

The extra term $\alpha \delta_{0}$ is only necessary in the case when $\mu(0)>0$. It follows that we have $\psi=\psi^{\prime}+\alpha \delta_{0} \in L$ and, consequently, applying the commutator $x \Pi-\Pi x$ to the function $\psi$, that we also have

$$
\int_{\mathbb{R}} A(y) \psi(y) d \mu(y)=\int_{\mathbb{R}} B(y) \psi(y) d \mu(y)=0 .
$$

\subsection{Integrability of the Palm kernel.}

LEMMA 3.5. Let $q \in U$ be such that $\Pi(q, q) \neq 0$. Then the kernel of the operator $\Pi^{q}$ has the integrable form

$$
\Pi^{q}(x, y)=\frac{A^{q}(x) B^{q}(y)-A^{q}(y) B^{q}(x)}{x-y},
$$

where

$$
\begin{aligned}
A^{q}(x)= & \frac{A(x) B(q)-A(q) B(x)}{\sqrt{(A(q))^{2}+(B(q))^{2}}} ; \\
B^{q}(x)= & \frac{A(x) A(q)+B(x) B(q)}{\sqrt{(A(q))^{2}+(B(q))^{2}}} \\
& -\frac{\sqrt{(A(q))^{2}+(B(q))^{2}}(A(x) B(q)-A(q) B(x))}{\Pi(q, q)(x-q)} .
\end{aligned}
$$

ProOF. We first consider the case $A(q)=0, B(q) \neq 0$, in which

$$
\Pi(x, q)=\frac{A(x) B(q)}{x-q}
$$

and

$$
\Pi^{q}(x, y)=\Pi(x, y)-\frac{B(q)^{2} A(x) A(y)}{\Pi(q, q)(x-q)(y-q)}=\frac{A^{q}(x) B^{q}(y)-A^{q}(y) B^{q}(x)}{x-y}
$$


with

$$
A^{q}(x)=A(x), \quad B^{q}(x)=B(x)-\frac{B(q)^{2} A(x)}{\Pi(q, q)(x-q)},
$$

as desired. Recalling that in an integrable representation of a kernel, the functions $A$ and $B$ are defined up to a unimodular change of variables (8), we reduce the general case to the particular one by a rotation

$$
A(x) \rightarrow \frac{A(x) B(q)-A(q) B(x)}{\sqrt{(A(q))^{2}+(B(q))^{2}}} ; \quad B(x) \rightarrow \frac{A(x) A(q)+B(x) B(q)}{\sqrt{(A(q))^{2}+(B(q))^{2}}} .
$$

In the discrete case, we have a dual to Proposition 3.5.

PROPOSITION 3.6. Let $\Pi$ be a projection operator with an integrable kernel. Let $q \in D$ be such that $\Pi(q, q) \neq 1$. Then the kernel of the operator $\Pi^{\breve{q}}$ has integrable form

$$
\Pi^{\breve{q}}(x, y)=\frac{A^{\breve{q}}(x) B^{\breve{q}}(y)-A^{\breve{q}}(y) B^{\breve{q}}(x)}{x-y},
$$

where $A^{\breve{q}}(q)=B^{\breve{q}}(q)=0$ and for $x \neq q, y \neq q$ we have

$$
\begin{aligned}
A^{\breve{q}}(x)= & \frac{A(x) B(q)-A(q) B(x)}{\sqrt{(A(q))^{2}+(B(q))^{2}}} ; \\
B^{\breve{q}}(x)= & \frac{A(x) A(q)+B(x) B(q)}{\sqrt{(A(q))^{2}+(B(q))^{2}}} \\
& +\frac{\sqrt{(A(q))^{2}+(B(q))^{2}}(A(x) B(q)-A(q) B(x))}{(1-\Pi(q, q))(x-q)} .
\end{aligned}
$$

PROOF. Direct substitution of (63) into (46).

\section{Multiplicative functionals and regularization.}

4.1. Outline of the section. The multiplicative functional corresponding to the function $\left(\frac{x-p}{x-q}\right)^{2}$ might diverge (as happens, for instance, for the Airy kernel), and in this section we describe a general scheme of regularization of multiplicative functionals. Our starting point is the formula (40), and we show that, under certain additional assumptions, the regularized multiplicative functional $\bar{\Psi}_{g}$ can still be defined if the multiplicative functional itself diverges, much in the same way in which the Hilbert-Carleman regularization of the Fredholm determinant is defined, cf. Simon [28]. Additional technical difficulties arise because we must consider 
functions bounded away neither from zero nor from infinity. In the following 9 subsections, we go back to a general phase space $(E, \mu)$ and a general locally trace class operator $\Pi$ of orthogonal projection onto a closed subspace $L \subset L_{2}(E, \mu)$. We use neither integrability nor Assumption 2. The main results of the section are Proposition 4.2 giving the existence of regularized multiplicative functionals and Proposition 4.3 establishing their continuous dependence on the function. Our first step is the regularization of additive functionals, for which we rely on the slow growth of variance for determinantal point processes (cf. e.g., [6]).

4.2. Regularization of additive functionals. Let $f: E \rightarrow \mathbb{C}$ be a Borel function. We set $S_{f}$ to be the corresponding additive functional, and, if $S_{f} \in$ $L_{1}\left(\operatorname{Conf}(E), \mathbb{P}_{\Pi}\right)$, then we set

$$
\bar{S}_{f}=S_{f}-\mathbb{E} S_{f}
$$

The random variable $\bar{S}_{f}$ will be called the normalized additive functional corresponding to $f$. We shall now see that the normalized additive functional can be defined even when the additive functional itself is not well defined. Set

$$
\operatorname{Var}(\Pi, f)=\frac{1}{2} \int_{E} \int_{E}|f(x)-f(y)|^{2}|\Pi(x, y)|^{2} d \mu(x) d \mu(y) .
$$

Note that the value $\operatorname{Var}(\Pi, f)$ does not change if the function $f$ is changed by an additive constant. If $S_{f} \in L_{2}\left(\operatorname{Conf}(E), \mathbb{P}_{\Pi}\right)$, then $\operatorname{Var}(\Pi, f)<+\infty$ and

$$
\operatorname{Var}\left(S_{f}\right)=\mathbb{E}\left|\bar{S}_{f}\right|^{2}=\operatorname{Var}(\Pi, f) .
$$

Note also the clear inequality

$$
\operatorname{Var}(\Pi, f) \leq 2 \int_{E}|f(x)|^{2} \Pi(x, x) d \mu(x)
$$

which is obtained by summing the inequality $|f(x)-f(y)|^{2} \leq 2\left(|f(x)|^{2}+\right.$ $|f(y)|^{2}$ ) over all $x, y$ and using the Pythagoras theorem (reproducing property of $\Pi$ on the diagonal):

$$
\Pi(x, x)=\int_{E}|\Pi(x, y)|^{2} d \mu(y) .
$$

The integral defining the variance of an additive functional may converge even when the integral defining its expectation does not: for instance, if $f(x)=(|x|+$ $1)^{-1}$ and $\Pi$ is the discrete sine-kernel. The normalized additive functional can nonetheless by continuity be defined in $L_{2}$ even when the additive functional itself diverges almost surely.

Introduce the Hilbert space $\mathcal{V}(\Pi)$ in the following way: the elements of $\mathcal{V}(\Pi)$ are functions $f$ on $E$ satisfying $\operatorname{Var}(\Pi, f)<+\infty$; functions that differ by a constant are identified, but, slightly abusing terminology we still refer to elements of $\mathcal{V}(\Pi)$ as functions. The square of the norm of an element $f \in \mathcal{V}(\Pi)$ is precisely $\operatorname{Var}(\Pi, f)$. By definition, bounded functions that are identically zero in 
the complement of a bounded set form a dense subset of $\mathcal{V}(\Pi)$. The correspondence $f \rightarrow \bar{S}_{f}$ is thus an isometric embedding of a dense subset of $\mathcal{V}(\Pi)$ into $L_{2}\left(\operatorname{Conf}(E), \mathbb{P}_{\Pi}\right)$; it therefore admits a unique isometric extension onto the whole space $\mathcal{V}(\Pi)$, and we obtain the following proposition.

PROPOSITION 4.1. There exists a unique linear isometric embedding

$$
\bar{S}: \mathcal{V}(\Pi) \rightarrow L_{2}\left(\operatorname{Conf}(E), \mathbb{P}_{\Pi}\right), \quad \bar{S}: f \rightarrow \bar{S}_{f}
$$

such that

1. $\mathbb{E} \bar{S}_{f}=0$ for all $f \in \mathcal{V}(\Pi)$;

2. if $S_{f} \in L_{1}\left(\operatorname{Conf}(E), \mathbb{P}_{\Pi}\right)$, then $\bar{S}_{f}$ is given by (64).

4.3. Regularization of multiplicative functionals. Given a function $g$ such that $\operatorname{Var}(\Pi, \log g)<+\infty$, set

$$
\widetilde{\Psi}_{g}=\exp \left(\bar{S}_{\log g}\right)
$$

By definition, we have

$$
\widetilde{\Psi}_{g_{1} g_{2}}=\widetilde{\Psi}_{g_{1}} \widetilde{\Psi}_{g_{2}} .
$$

Since $\mathbb{E} \bar{S}_{\log g}=0$, by Jensen's inequality, for any positive function $g$ we have

$$
\mathbb{E} \tilde{\Psi}_{g} \geq 1 \text {. }
$$

The expectation $\mathbb{E} \widetilde{\Psi}_{g}$ may however be infinite, and our next aim is to give conditions for its finiteness.

It will be convenient for us to allow zero values for the function $g$ : let therefore $g$ be nonnegative, set $E_{0}=\{x \in E: g(x)=0\}$, assume that the subset $\operatorname{Conf}\left(E ; E \backslash E_{0}\right)$ of those configurations all whose particles lie in $E \backslash E_{0}$ has positive probability, consider the restriction of our measure $\mathbb{P}$ onto the subspace $\operatorname{Conf}\left(E ; E \backslash E_{0}\right)$, introduce the corresponding functional $\widetilde{\Psi}_{g}$ and extend it to the whole of $E$ by setting $\widetilde{\Psi}_{g}(X)=0$ for all configurations containing a particle at $E_{0}$. Assume that tr $\chi_{E_{0}} \Pi \chi_{E_{0}}<+\infty$. Then we have $\mathbb{P}_{\Pi}\left(\operatorname{Conf}\left(E ; E \backslash E_{0}\right)\right)=$ $\operatorname{det}\left(1-\chi_{E_{0}} \Pi \chi_{E_{0}}\right)$. The operator $\chi_{E_{0}} \Pi \chi_{E_{0}}$ is positive, contractive and has finite trace, so the operator $1-\chi_{E_{0}} \Pi \chi_{E_{0}}$ is invertible once it is injective. Thus, if a function $\varphi \in L$ satisfying $\varphi(x)=0$ for all $x \in E \backslash E_{0}$ must be the zero function, then $\mathbb{P}_{\Pi}\left(\operatorname{Conf}\left(E ; E \backslash E_{0}\right)\right)>0$.

If $\widetilde{\Psi}_{g} \in L_{1}\left(\operatorname{Conf}(E), \mathbb{P}_{\Pi}\right)$, then, as before, we write

$$
\bar{\Psi}_{g}=\frac{\widetilde{\Psi}_{g}}{\mathbb{E} \widetilde{\Psi}_{g}} .
$$

The main result of this section is the following proposition.

PROPOSITION 4.2. Let $g$ be a nonnegative function satisfying the following: 
1. there exist $\varepsilon>0, M>\varepsilon$ such that the set $E^{\varepsilon, M}=\{x \in E: g(x)<$ $\varepsilon$ or $g(x)>M\}$ is bounded and

$$
\left\|\chi_{E^{\varepsilon, M}} \Pi\right\|<1
$$

2.

$$
\int_{E^{\varepsilon, M}}|g(x)| \Pi(x, x) d \mu(x)+\int_{E \backslash E^{\varepsilon, M}}|g(x)-1|^{2} \Pi(x, x) d \mu(x)<+\infty .
$$

Then $\widetilde{\Psi}_{g} \in L_{1}\left(\operatorname{Conf}(E), \mathbb{P}_{\Pi}\right)$, the subspace $\sqrt{g} L$ is closed, the corresponding operator of orthogonal projection $\Pi^{g}$ is locally of trace class, and we have

$$
\mathbb{P}_{\Pi g}=\bar{\Psi}_{g} \mathbb{P}_{\Pi} .
$$

We also need to establish continuity of $\bar{\Psi}_{g}$ as a function of $g$. This continuity is established in a specially constructed function space whose definition is a bit involved. We fix positive numbers $\alpha>0, \varepsilon>0, M>\varepsilon$ and two bounded Borel subsets $B^{1}, B^{2}$ of $E$ satisfying

$$
\left\|\chi_{B^{1} \cup B^{2}} \Pi\right\|<1 .
$$

We now let $\mathscr{G}$ be the set of nonnegative measurable functions $g$ on $E$ satisfying

1. $\{x: g(x)<\varepsilon\} \subset B^{1}$;

2. $\{x: g(x)>M\} \subset B^{2}$;

3. $\int_{B^{2}}|g(x)|^{1+\alpha} \Pi(x, x) d \mu(x)+\int_{E \backslash B^{2}}|g(x)-1|^{2} \Pi(x, x) d \mu(x)<+\infty$.

We metrize the set $\mathscr{G}$ by setting

$$
\begin{aligned}
d_{\mathscr{G}}\left(g_{1}, g_{2}\right)= & \int_{B^{2}}\left|g_{1}(x)-g_{2}(x)\right|^{1+\alpha} \Pi(x, x) d \mu(x) \\
& +\int_{E \backslash B^{2}}\left|g_{1}(x)-g_{2}(x)\right|^{2} \Pi(x, x) d \mu(x) .
\end{aligned}
$$

The distance $d_{\mathscr{G}}$ turns $\mathscr{G}$ into a complete separable metric space.

PROPOSITION 4.3. For any $\alpha^{\prime}: 0<\alpha^{\prime}<\alpha$, the correspondences $g \rightarrow \widetilde{\Psi}_{g}$, $g \rightarrow \bar{\Psi}_{g}$ induce continuous mappings from $\mathscr{G}$ to $L_{1+\alpha^{\prime}}\left(\operatorname{Conf}(E), \mathbb{P}_{\Pi}\right)$,

REMARK. Along similar lines, one also proves that the correspondence $g \rightarrow$ $\Pi^{g}$ induces a continuous mapping from the space $\mathscr{G}$ to $\mathscr{I}_{1, \text { loc }}(E, \mu)$.

Propositions 1.3, 1.5 directly follow from Propositions 4.2, 4.3.

The proof of the main Propositions 4.2 and 4.3 occupies the bulk of this section. We separately consider the "main part" (where the function $g$ is bounded away from 0 and $\infty$ ), the neighbourhood of 0 and the neighbourhood of $\infty$. The proof is 
concluded by decomposing a general function $g$ as a product of these three types of functions.

First, we reduce ourselves to the case of positive $g$. Let $E_{0}=\{x: g(x)=0\}$. Our assumptions imply $\mathbb{P}_{\Pi}\left(\operatorname{Conf}\left(E ; E \backslash E_{0}\right)\right)>0$ and so, restricting ourselves, if necessary, to the subset $\operatorname{Conf}\left(E ; E \backslash E_{0}\right)$, we can assume that the function $g$ is positive.

REMARK. Our aim is to apply Proposition 4.2 to functions of the form

$$
g(x)=\left(\frac{\left(x-p_{1}\right) \cdots\left(x-p_{l}\right)}{\left(x-q_{1}\right) \cdots\left(x-q_{l}\right)}\right)^{2} .
$$

In the continuous case, such functions are almost surely nonzero. In the discrete case, however, the finite zero set of our function has positive measure: whence the need for the extra subset $E_{0}$ in our proposition, and the need of the assumption that the subspace $L$ not admit finitely-supported functions in the formulation of Theorem 1.6.

4.4. An estimate of diagonal values of the kernel $\Pi^{g}$. Iterating multiplicative functionals, we need to estimate traces of the resulting kernels. The following simple proposition shows that diagonal values of the kernel of $\Pi^{g}$ can be estimated from above by the diagonal values of the kernel $\Pi$.

PROPOSITION 4.4. Let the kernel $\Pi$ satisfy Assumption 1, and let $g$ be a nonnegative bounded function on $E$ such that the operator $1+(g-1) \Pi$ is invertible. Then for any $q \in U$ we have

$$
\Pi^{g}(q, q) \leq g(q)\left\|(1+(g-1) \Pi)^{-1}\right\| \Pi(q, q) .
$$

ProOF. As before, we let $\langle$,$\rangle be the standard inner product in L_{2}(E, \mu)$ and we write $v_{q}(x)=\Pi(x, q)$ so that $\Pi(q, q)=\left\langle v_{q}, v_{q}\right\rangle$. By definition then

$$
\begin{aligned}
\Pi^{g}(q, q) & =g(q)\left\langle\Pi(1+(g-1) \Pi)^{-1} v_{q}, v_{q}\right\rangle \\
& \leq g(q)\left\|(1+(g-1) \Pi)^{-1}\right\|\left\langle v_{q}, v_{q}\right\rangle
\end{aligned}
$$

4.5. The case of functions $g$ bounded away from 0 and $\infty$. Let $\mathscr{A}_{2}(\Pi)$ be the set of positive Borel functions $g$ on $E$ satisfying

1. $0<\inf _{E} g \leq \sup _{E} g<\infty$;

2 .

$$
\int_{E}|g(x)-1|^{2} \Pi(x, x) d \mu(x)<+\infty .
$$


By definition, the set $\mathscr{A}_{2}(\Pi)$ is a semigroup under multiplication.

Endow the set $\mathscr{A}_{2}(\Pi)$ with a metric by setting the distance between two functions $g_{1}$ and $g_{2}$ to be

$$
\sqrt{\int_{E}\left|g_{1}(x)-g_{2}(x)\right|^{2} \Pi(x, x) d \mu(x)} .
$$

Using the second condition in the definition of $\mathscr{A}_{2}(\Pi)$ and the estimate (66), for any $g \in \mathscr{A}_{2}(\Pi)$ we have

$$
\operatorname{Var}(\Pi, g-1)<+\infty \text {. }
$$

Since on any interval of the positive half-line, bounded away from zero and infinity, the quantity $|\log t-t+1| / t^{2}$ is bounded both above and below, for any function $g \in \mathscr{A}_{2}(\Pi)$, we also have

$$
\operatorname{Var}(\Pi, \log g)<+\infty .
$$

In particular, for any function $g \in \mathscr{A}_{2}(\Pi)$ the functional $\widetilde{\Psi}_{g}$ is well defined. The following proposition, the main result of this subsection, establishes its integrability.

Proposition 4.5. For any $p \geq 1$, and any function $g \in \mathscr{A}_{2}(\Pi)$ we have $\widetilde{\Psi}_{g} \in$ $L_{p}\left(\operatorname{Conf}(E), \mathbb{P}_{\Pi}\right)$. The correspondences

$$
g \rightarrow \widetilde{\Psi}_{g}, \quad g \rightarrow \bar{\Psi}_{g}
$$

are continuous mappings from $\mathscr{A}_{2}(\Pi)$ to $L_{p}\left(\operatorname{Conf}(E), \mathbb{P}_{\Pi}\right)$.

PROOF. Let

$$
\mathscr{A}_{2}^{\varepsilon, M}(\Pi)=\left\{g \in \mathscr{A}_{2}(\Pi): \varepsilon \leq \inf _{E} g \leq \sup _{E} g \leq M\right\} .
$$

Proposition 4.6. For any $\varepsilon>0, M>0$ there exists a constant $C_{\varepsilon, M}>0$ such that any $g \in \mathscr{A}_{2}^{\varepsilon, M}$ (П) satisfies

$$
\log \mathbb{E}\left|\widetilde{\Psi}_{g}\right|^{2} \leq C_{\varepsilon, M} \int_{E}|g(x)-1|^{2} \Pi(x, x) d \mu(x) .
$$

PROOF. It suffices to prove the estimate

$$
\log \mathbb{E} \widetilde{\Psi}_{g} \leq C_{\varepsilon, M} \int_{E}|g(x)-1|^{2} \Pi(x, x) d \mu(x),
$$

and (71) follows by multiplicativity (67). It suffices to establish (72) in the case when the set $\{x \in E: g(x) \neq 1\}$ is bounded, as the general case follows by Fatou's lemma. Now there exists a constant $C_{2}>0$ such that

$$
\begin{aligned}
\log \mathbb{E} \Psi_{g} & \leq \operatorname{tr}(\sqrt{g-1} \Pi \sqrt{g-1})+C_{2}\|\sqrt{g-1} \Pi \sqrt{g-1}\|_{2}^{2} \\
& =\int_{E}(g(x)-1) \Pi(x, x) d \mu(x)+C_{2} \int_{E}|g(x)-1|^{2} \Pi(x, x) d \mu(x) .
\end{aligned}
$$


We have assumed boundedness of the set $\{x \in E: g(x) \neq 1\}$ in order that the integral $\int_{E}(g(x)-1) \Pi(x, x) d \mu(x)$ be well defined; it will, however, disappear from the final result. From (70), again using the fact that the quantity $\mid \log t-t+$ $1 \mid / t^{2}$ is bounded both above and below by constants only depending on $\varepsilon$ and $M$, we obtain

$$
\begin{aligned}
& \left|\int_{E}(g(x)-1) \Pi(x, x) d \mu(x)-\int_{E} \log g(x) \Pi(x, x) d \mu(x)\right| \\
& \quad \leq C_{\varepsilon, M} \int_{E}|g(x)-1|^{2} \Pi(x, x) d \mu(x),
\end{aligned}
$$

whence finally

$$
\log \mathbb{E} \widetilde{\Psi}_{g}=\log \mathbb{E} \Psi_{g}-\mathbb{E} S_{\log g} \leq C_{\varepsilon, M}^{\prime} \int_{E}|g(x)-1|^{2} \Pi(x, x) d \mu(x)
$$

Proposition 4.7. For any $\varepsilon>0, M>0$ there exists a constant $C_{\varepsilon, M}>0$ such that for $g_{1}, g_{2} \in \mathscr{A}_{2}^{\varepsilon, M}(\Pi)$ we have

$$
\mathbb{E}\left|\widetilde{\Psi}_{g_{1}}-\widetilde{\Psi}_{g_{2}}\right| \leq \mathbb{E}\left|\widetilde{\Psi}_{g_{1}}\right|^{2}\left(\exp \left(C_{\varepsilon, M} \int_{E}\left|g_{1}(x)-g_{2}(x)\right|^{2} \Pi(x, x) d \mu(x)\right)-1\right) .
$$

For any $p \geq 1$, the correspondence $g \rightarrow \widetilde{\Psi}_{g}$ induces a continuous mapping from $\mathscr{A}_{2}^{\varepsilon, M}$ (П) to $L_{p}\left(\operatorname{Conf}(E), \mathbb{P}_{\Pi}\right)$.

PROOF. Since $\mathbb{E} \widetilde{\Psi}_{g} \geq 1$, we have

$$
\mathbb{E}\left|\tilde{\Psi}_{g}-1\right|^{2} \leq \mathbb{E} \tilde{\Psi}_{g^{2}}-1
$$

From the estimate (71), we have

$$
\mathbb{E}\left|\widetilde{\Psi}_{g}-1\right|^{2} \leq \exp \left(C_{\varepsilon, M} \int_{E}|g(x)-1|^{2} \Pi(x, x) d \mu(x)\right)-1 .
$$

Applying (75) to $g=g_{1} / g_{2}$, recalling the boundedness of both $g_{1}$ and $g_{2}$ and using multiplicativity, we obtain the proposition. Since, for any $p \geq 1$, we have $\widetilde{\Psi}_{g} p=$ $\left(\widetilde{\Psi}_{g}\right)^{p}$, and $\left(\mathscr{A}_{2}^{\varepsilon, M}(\Pi)\right)^{p} \subset \mathscr{A}_{2}^{\varepsilon^{p}, M^{p}}(\Pi)$, the desired continuity in $L_{p}$ follows as well.

Proposition 4.7 implies Proposition 4.5.

4.6. The Radon-Nikodym derivative.

COROLlARY 4.8. Let $g \in \mathscr{A}_{2}(\Pi)$ be such that the operator $1+(g-1) \Pi$ is invertible. Then the operator $\Pi^{g}$ is locally of trace class, and we have

$$
\mathbb{P}_{\Pi g}=\bar{\Psi}_{g} \mathbb{P}_{\Pi}
$$


ProOF. Let $E^{(n)}$ be a sequence of bounded sets exhausting $E$, set $g_{n}=1+$ $(g-1) \chi_{E^{(n)}}$, and note that $\left\|\left(g_{n}-g\right) \Pi\right\| \rightarrow 0$ as $n \rightarrow \infty$. For any $n$, we have

$$
\mathbb{P}_{\Pi g_{n}}=\bar{\Psi}_{g_{n}} \mathbb{P}_{\Pi}
$$

The operators $\Pi^{g}=\sqrt{g} \Pi(1+(g-1) \Pi)^{-1} \sqrt{g}, \Pi^{g_{n}}=\sqrt{g_{n}} \Pi\left(1+\left(g_{n}-\right.\right.$ 1) $\Pi)^{-1} \sqrt{g_{n}}$ are locally of trace class since so is $\Pi$. Proposition 2.6 implies that $\Pi^{g_{n}} \rightarrow \Pi^{g}$ in $\mathscr{I}_{1, \mathrm{loc}}(E, \mu)$, and Proposition 2.2 implies that, as $n \rightarrow \infty$, the sequence of measures $\mathbb{P}_{\Pi^{g} n}$ weakly converges to $\mathbb{P}_{\Pi^{g}}$ in the space of probability measures on $\operatorname{Conf}(E)$. Proposition 4.5 implies $\bar{\Psi}_{g_{n}} \rightarrow \bar{\Psi}_{g}$ in $L_{1}\left(\operatorname{Conf}(E), \mathbb{P}_{\Pi}\right)$, whence $\bar{\Psi}_{g_{n}} \mathbb{P}_{\Pi} \rightarrow \bar{\Psi}_{g} \mathbb{P}_{\Pi}$ weakly in the space of probability measures on Conf $(E)$, implying (76).

\subsection{Multiplicative functionals corresponding to a function $g \geq 1$.}

4.7.1. The case of bounded $g$. Proposition 2.7 takes a simpler form when our bounded function $g$ satisfies $g \geq 1$. First, in this case the subspace $\sqrt{g} L$ is automatically closed. Second, if $\sqrt{g-1} \Pi \sqrt{g-1}$ belongs to the trace class, then the operator $1+(g-1) \Pi$ is automatically invertible. To verify this, observe first that in this case the operator $\sqrt{g-1} \Pi$ is Hilbert-Schmidt, consequently, the operator $(g-1) \Pi$ is also Hilbert-Schmidt and, a fortiori, compact. To check the invertibility of the operator $1+(g-1) \Pi$, it thus suffices to check its injectivity, that is, to prove that a function $\varphi$ satisfying

$$
\varphi+(g-1) \Pi \varphi=0
$$

must be the zero function. Set $\psi=-\sqrt{g-1} \Pi \varphi$ so that $\varphi=\sqrt{g-1} \psi$. By definition, both $\varphi$ and $\psi$ are zero on the set $\{x \in E: g(x)=1\}$. From (77) we now have

$$
\psi+\sqrt{g-1} \Pi \sqrt{g-1} \psi=0
$$

whence

$$
\langle\psi, \psi\rangle+\langle\Pi \varphi, \varphi\rangle=0
$$

whence finally $\varphi=\psi=0$.

We can now reformulate Proposition 2.7 in the following simpler form.

PROPOSITION 4.9. Let $g$ be a bounded measurable function on $E$ satisfying $g \geq 1$ and such that the operator $\sqrt{g-1} \Pi \sqrt{g-1}$ belongs to the trace class. Then all the conclusions of Proposition 2.7 hold for the function $g$. 
4.7.2. The case of unbounded $g$. The function $(x-p) /(x-q)$ is unbounded on $\mathbb{R}$, and we prepare, for future use, a proposition on multiplicative functionals corresponding to unbounded functions. As before, we start with a locally traceclass operator $\Pi$ of orthogonal projection onto a subspace $L$. We consider a function $g \geq 1$ such that the space $\sqrt{g} L$ is a subspace of $L_{2}(E, \mu)$; since $g \geq 1$, it is automatically a closed subspace, and we let $\Pi^{g}$ be the operator of orthogonal projection onto $\sqrt{g} L$.

Proposition 4.10. Let $\alpha>0$ and let $g$ be a Borel function on $E$ satisfying $g>1+\alpha$. Assume that

1. we have $\sqrt{g} L \subset L_{2}(E, \mu)$;

2. the operator $\sqrt{g-1} \Pi$ is Hilbert-Schmidt.

Then $\Psi_{g} \in L_{1}\left(\operatorname{Conf}(E), \mathbb{P}_{\Pi}\right)$, and we have

$$
\frac{\Psi_{g} \mathbb{P}_{\Pi}}{\int_{\operatorname{Conf}(E)} \Psi_{g} d \mathbb{P}_{\Pi}}=\mathbb{P}_{\Pi^{g}} .
$$

ProOF. For $R>0$ set $g^{R}(x)=g(x)$ if $g(x)<R$ and $g^{R}(x)=1$ otherwise. As in Section 2, we use the notation $\Pi_{g}=\sqrt{g-1} \Pi \sqrt{g-1}$.

The Hilbert-Schmidt norm of the operator $\sqrt{g-1} \Pi$ is given by the formula

$$
\|\sqrt{g-1} \Pi\|_{2}=\int_{E}(g(x)-1) \Pi(x, x) d \mu(x) .
$$

For $R_{1}>R_{2}>0$, we have

$$
\begin{aligned}
& \left\|\sqrt{g^{R_{1}}-1} \Pi-\sqrt{g^{R_{2}}-1} \Pi\right\|_{2} \\
& \quad=\int_{E}\left(\sqrt{g^{R_{1}}(x)-1}-\sqrt{g^{R_{2}}(x)-1}\right)^{2} \Pi(x, x) d \mu(x),
\end{aligned}
$$

and, in view of the convergence of the integral in (79), the right-hand side of (80) becomes arbitrarily small once $R_{1}, R_{2}$ are sufficiently large. It follows that, as $R \rightarrow \infty$, the sequence of operators $\sqrt{g^{R}-1} \Pi$ converges in Hilbert-Schmidt norm, and, consequently, the sequence of operators $\Pi_{g R}$ converges in the traceclass norm. Write $\Pi_{g}=\lim _{R \rightarrow \infty} \Pi_{g R}$, and note that trace-class convergence implies

$$
\operatorname{det}\left(1+\Pi_{g}\right)=\lim _{R \rightarrow \infty} \operatorname{det}\left(1+\Pi_{g R}\right) .
$$

For any $X$, as $R$ grows, the sequence $\Psi_{g}(X)$ increases (possibly assuming infinite value starting from some $R$ ). By definition of the multiplicative functional, we have pointwise convergence

$$
\Psi_{g}=\lim _{R \rightarrow \infty} \Psi_{g},
$$


and, by (81), this convergence also takes place in $L_{1}\left(\operatorname{Conf}(E), \mathbb{P}_{\Pi}\right)$. We thus finally have

$$
\int_{\operatorname{Conf}(E)} \Psi_{g} d \mathbb{P}_{\Pi}=\operatorname{det}\left(1+\Pi_{g}\right)
$$

Using (43), write

$$
\Pi^{g^{R}}=\sqrt{1+\left(g^{R}-1\right)^{-1}} \Pi_{g^{R}}\left(1+\Pi_{g^{R}}\right)^{-1} \sqrt{1+\left(g^{R}-1\right)^{-1}} .
$$

It follows that the sequence of operators $\Pi^{g^{R}}$ converges, as $R \rightarrow \infty$, in the space of locally trace-class operators. Since $\Pi^{g^{R}}$ is the operator of orthogonal projection onto the subspace $\sqrt{g^{R}} L$, we obtain that

$$
\Pi^{g}=\lim _{R \rightarrow \infty} \Pi^{g^{R}} \quad \text { in } \mathscr{I}_{1, \mathrm{loc}}(E, \mu) .
$$

Now, for any fixed $R$, by Proposition 4.9 , we have

$$
\frac{\Psi_{g^{R}} \mathbb{P}_{\Pi}}{\int_{\operatorname{Conf}(E)} \Psi_{g^{R}} d \mathbb{P}_{\Pi}}=\mathbb{P}_{\Pi^{g} R} .
$$

Proposition 2.2 implies that the sequence of measures $\mathbb{P}_{\Pi^{g} R}$ weakly converges to $\mathbb{P}_{\Pi^{g}}$ as $R \rightarrow \infty$, and (84), together with (82) and (83), implies the desired relation (78).

\subsection{Conclusion of the proof of Proposition 4.2. Set}

$$
\begin{aligned}
& g_{0}=(g-1) \chi_{E \backslash E^{\varepsilon, M}}+1 . \\
& g_{1}=(g-1) \chi_{\{x \in E: g(x)<\varepsilon\}}+1 . \\
& g_{2}=(g-1) \chi_{\{x \in E: g(x)>M\}}+1 .
\end{aligned}
$$

By definition, $g=g_{0} g_{1} g_{2}$.

By definition, the subspace $\sqrt{g_{0}} L$ is closed, and, by Corollary 4.8 , we have $\mathbb{P}_{\Pi^{g_{0}}}=\bar{\Psi}_{g_{0}} \mathbb{P}_{\Pi}$. Proposition 4.4 implies the existence of a positive constant $C$ such that $\Pi^{g_{0}}(x, x) \leq C \Pi(x, x)$ for $\mu$-amost all $x \in E$. Applying Proposition 2.10 to the function $g_{1}$ and the operator $\Pi^{g_{0}}$, we arrive at the formula

$$
\mathbb{P}_{\Pi^{g_{1} g_{0}}}=\bar{\Psi}_{g_{1}} \mathbb{P}_{\Pi^{g_{0}}}=\bar{\Psi}_{g_{1} g_{0}} \mathbb{P}_{\Pi} .
$$

Again, Proposition 4.4 implies the existence of a positive constant $C$ such that $\Pi^{g_{1} g_{0}}(x, x) \leq C \Pi(x, x)$ for $\mu$-amost all $x \in E$, whence

$$
\int_{E}\left|g_{2}(x)-1\right| \Pi^{g_{1} g_{0}}(x, x) d \mu(x)<+\infty,
$$

and, consequently, that the operator $\sqrt{g_{2}-1} \Pi^{g_{1}} g_{0}$ is Hilbert-Schmidt. 
We therefore apply Proposition 4.10 to the function $g_{2}$ and the operator $\Pi^{g_{1}} g_{0}$ and obtain

$$
\mathbb{P}_{\Pi^{g_{2} g_{1} g_{0}}}=\bar{\Psi}_{g_{2}} \mathbb{P}_{\Pi^{g_{1} g_{0}}} .
$$

Observe that we only used regularized multiplicative functionals at the very first step of our argument. In other words, there exist constants $C_{1}, C_{2}, C_{3}$ such that we have

$$
\begin{aligned}
\mathbb{P}_{\Pi^{g_{0}}} & =C_{0} \widetilde{\Psi}_{g_{0}} \mathbb{P}_{\Pi}, \quad \mathbb{P}_{\Pi^{g_{1} g_{0}}}=C_{1} \Psi_{g_{1}} \mathbb{P}_{\Pi^{g_{0}}}, \\
\mathbb{P}_{\Pi^{g}} & =\mathbb{P}_{\Pi^{g_{2} g_{1} g_{0}}}=C_{2} \Psi_{g_{2}} \mathbb{P}_{\Pi^{g_{1} g_{0}}} .
\end{aligned}
$$

By definition, we have $\widetilde{\Psi}_{g}=\widetilde{\Psi}_{g_{2}} \widetilde{\Psi}_{g_{1}} \widetilde{\Psi}_{g_{0}}$ and, consequently, for a suitable positive constant $C^{\prime}$, also $\widetilde{\Psi}_{g}=C^{\prime} \widetilde{\Psi}_{g_{0}} \Psi_{g_{1}} \Psi_{g_{2}}$. Passing from $\widetilde{\Psi}_{g}$ to $\bar{\Psi}_{g}$ and noting that the Radon-Nikodym derivative must by definition have expectation 1 , we finally obtain the desired relation $\mathbb{P}_{\Pi g}=\bar{\Psi}_{g} \mathbb{P}_{\Pi}$.

4.9. Proof of Proposition 4.3. Set $\mathscr{G}^{0}=\left\{g \in \mathscr{G}:\left(g_{0}-1\right) \chi_{E \backslash\left(B^{1} \cup B^{2}\right)}=g_{0}-1\right\}$ and endow the set $\mathscr{G}^{0}$ with topology induced from $\mathscr{G}$, or, equivalently, from $L_{2}(E, \mu)$. Set $\mathscr{G}^{1}=\left\{g_{1} \in \mathscr{G}:\left(g_{1}-1\right) \chi_{B^{1}}=g_{1}-1\right\}$, and endow the set $\mathscr{G}^{1}$ with topology induced from $\mathscr{G}$, or, equivalently, from $L_{2}(E, \mu)$ or, equivalently again, from $L_{1}(E, \mu)$ (recall here that our functions are uniformly bounded). Finally, set $\mathscr{G}^{2}=\left\{g \in \mathscr{G}:\left(g_{2}-1\right) \chi_{B^{2}}=g_{2}-1\right\}$ endow the set $\mathscr{G}^{2}$ with topology induced from $\mathscr{G}$, or, equivalently, from $L_{1+\alpha}(E, \mu)$. The continuity of the mapping $g_{0} \rightarrow \widetilde{\Psi}_{g_{0}}$ from $\mathscr{G}^{0}$ to $L_{p}(E, \mu)$ directly follows from Propositions 4.5, 4.7. Next, the correspondence $g_{1} \rightarrow \widetilde{\Psi}_{g_{1}}$ is a continuous mapping from $\mathscr{G}^{1}$ to $L_{p}(E, \mu)$ by bounded convergence theorem. The correspondence $g_{2} \rightarrow \Psi_{g_{2}}$ induces a continuous mapping from the space $\mathscr{G}^{2}$ to $L_{1+\alpha}(E, \mu)$ by the dominated convergence theorem. Setting $g=g_{0} g_{1} g_{2}$, using the Hoelder inequality (and keeping in mind that $p$ can be taken arbitrarily large), we obtain the desired continuity of the correspondences $g \rightarrow \widetilde{\Psi}_{g}, g \rightarrow \bar{\Psi}_{g}$ considered as mappings from $\mathscr{G}$ to $L_{1+\alpha^{\prime}}(E, \mu)$.

4.10. Proof of Proposition 1.3. Let $l \in \mathbb{N}$ and take distinct points $p_{1}, \ldots, p_{l}$, $q_{1}, \ldots, q_{l} \in U$. We check that one can choose $\varepsilon>0$ small enough in such a way that the function

$$
g(x)=\left(\frac{\left(x-p_{1}\right) \cdots\left(x-p_{l}\right)}{\left(x-q_{1}\right) \cdots\left(x-q_{l}\right)}\right)^{2}
$$

satisfies the assumptions of Proposition 4.3 with an arbitrary $\alpha<1 / 2$ and $B^{1}=$ $\left\{x \in \mathbb{R}: \max _{i=1, \ldots, l}\left|x-p_{i}\right|<\varepsilon\right\}, B^{2}=\left\{x \in \mathbb{R}: \max _{i=1, \ldots, l}\left|x-q_{i}\right|<\varepsilon\right\}$. In this proof, $C$ stands for a constant depending only on $q_{1}, \ldots, q_{l}$ and $\varepsilon$. The kernel $\Pi$ is smooth, and if $\left|x-q_{i}\right|<\varepsilon$, then $\left|\Pi^{q_{1}, \ldots, q_{l}}(x, x)\right|<C\left|x-q_{i}\right|^{2}$, whence for any $\alpha<1 / 2$ we have

$$
\int_{B^{2}}|g(x)|^{1+\alpha} \Pi^{q_{1}, \ldots, q_{l}}(x, x) d \mu(x)<+\infty .
$$


For any $\varepsilon>0$, on the set $\left\{x \in \mathbb{R}: \min _{i=1, \ldots, l}\left|x-q_{i}\right|>\varepsilon\right\}$ we have $|g(x)-1|^{2} \leq$ $C\left(1+x^{2}\right)^{-1}$, whence

$$
\int_{\left\{x \in \mathbb{R}: \min _{i=1, \ldots, l}\left|x-q_{i}\right|>\varepsilon\right\}}|g(x)-1|^{2} \Pi(x, x) d \mu(x)<+\infty .
$$

The operator $\Pi^{q_{1}, \ldots, q_{l}}$ is a finite-rank perturbation of the operator $\Pi$, whence

$$
\int_{\left\{x \in \mathbb{R}: \min _{i=1, \ldots, l}\left|x-q_{i}\right|>\varepsilon\right\}}|g(x)-1|^{2} \Pi^{q_{1}, \ldots, q_{l}}(x, x) d \mu(x)<+\infty .
$$

Proposition 4.3 now implies Proposition 1.3.

Proposition 4.2 together with Proposition 3.1 immediately imply the following.

COROLLARY 4.11. Under the assumptions of Theorem 1.4, for any distinct points $p_{1}, \ldots, p_{l}, q_{1}, \ldots, q_{l} \in U$, for the corresponding reduced Palm measures are equivalent, and we have

$$
\frac{d \mathbb{P}_{\Pi^{p_{1}, \ldots, p_{l}}}}{d \mathbb{P}_{\Pi^{q_{1}, \ldots, q_{l}}}}=\bar{\Psi}_{\left|\frac{\left(x-p_{1}\right) \cdots\left(x-p_{l}\right)}{\left(x-q_{1}\right) \cdots\left(x-q_{l}\right)}\right|^{2}} .
$$

Together with Proposition 2.19, Corollary 4.11 implies Theorem 1.4. Theorem 1.4 is proved completely.

4.11. Proof of Proposition 1.5. Denote $q_{i}=\sigma\left(p_{i}\right), i=1, \ldots, l$; of course, we have $\left\{p_{1}, \ldots, p_{l}\right\}=\left\{q_{1}, \ldots, q_{l}\right\}$. Set

$$
g(x)=\prod_{i=1}^{m}\left(\frac{x-q_{i}}{x-p_{i}}\right)^{2} \chi_{E \backslash\left\{p_{1}, \ldots, p_{l}\right\}}(x) .
$$

The function $g$ is bounded, and Assumption 4 implies

$$
\sum_{E}|g(x)-1|^{2}<+\infty
$$

and, since no nonzero function in $L$ is supported on a finite set, we conclude applying Proposition 4.5 to the function $g$ and the kernel $\Pi^{p_{1}, \ldots, p_{m}, \breve{p}_{m+1}, \ldots, \breve{p}_{l}}$.

In a similar way to the continuous case, Proposition 4.2 and Proposition 3.2 together imply that, under the assumptions of Proposition 1.5, we have

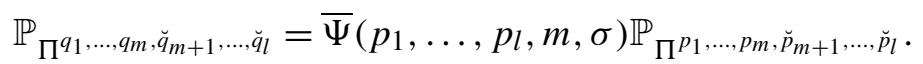

The relation (90) together with Proposition 2.19 implies Theorem 1.6.

Acknowledgments. Grigori Olshanski posed the problem to me and suggested that the Radon-Nikodym derivative be given by a multiplicative functional; I am greatly indebted to him. I am deeply grateful to Alexei Klimenko and Cosme Louart for useful discussions. I am deeply grateful to the anonymous referees whose suggestions have been of great help in improving the presentation.

Part of this work was done while I was visiting Institut Henri Poincaré in Paris and the Max Planck Institute in Bonn; I am deeply grateful to these institutions for their warm hospitality. 


\section{REFERENCES}

[1] Benjamini, I., Lyons, R., Peres, Y. and Schramm, O. (2001). Uniform spanning forests. Ann. Probab. 29 1-65. MR1825141

[2] Borodin, A., Okounkov, A. and Olshanski, G. (2000). Asymptotics of Plancherel measures for symmetric groups. J. Amer. Math. Soc. 13 481-515. MR1758751

[3] Borodin, A. and Olshanski, G. (2005). Random partitions and the gamma kernel. Adv. Math. 194 141-202. MR2141857

[4] Borodin, A. and Rains, E. M. (2005). Eynard-Mehta theorem, Schur process, and their Pfaffian analogs. J. Stat. Phys. 121 291-317. MR2185331

[5] Bufetov, A. I. (2012). On multiplicative functionals of determinantal processes. Uspekhi Mat. Nauk 67 177-178. Translation in Russian Math. Surveys 67 (2012) 181-182. MR2961470

[6] Bufetov, A. I. (2012). On the Vershik-Kerov conjecture concerning the ShannonMcMillan-Breiman theorem for the Plancherel family of measures on the space of Young diagrams. Geom. Funct. Anal. 22 938-975. MR2984121

[7] Bufetov, A. I. (2013). Infinite determinantal measures. Electron. Res. Announc. Math. Sci. 20 12-30. MR3035262

[8] BufETOV, A. I. (2015). On the action of the diffeomorphism group on determinantal measures. Uspekhi Mat. Nauk 70 175-176. Translation in Russian Math. Surveys 70 (2015) 953954. MR3438557

[9] Camilier, I. and DeCReusefond, L. (2010). Quasi-invariance and integration by parts for determinantal and permanental processes. J. Funct. Anal. 259 268-300. MR2610387

[10] Daley, D. J. and Vere-Jones, D. (2008). An Introduction to the Theory of Point Processes. Vol. II: General Theory and Structure, 2nd ed. Springer, New York. MR2371524

[11] Ghosh, S. Rigidity and tolerance in Gaussian zeros and Ginibre eigenvalues: Quantitative estimates. Available at arXiv:1211.3506.

[12] Ghosh, S. (2015). Determinantal processes and completeness of random exponentials: The critical case. Probab. Theory Related Fields 163 643-665. MR3418752

[13] Ghosh, S. and Peres, Y. (2017). Rigidity and tolerance in point processes: Gaussian zeros and Ginibre eigenvalues. Duke Math. J. 166 1789-1858. MR3679882

[14] Hough, J. B., Krishnapur, M., Peres, Y. and ViráG, B. (2006). Determinantal processes and independence. Probab. Surv. 3 206-229. MR2216966

[15] Its, A. R., Izergin, A. G., Korepin, V. E. and Slavnov, N. A. (1990). Differential equations for quantum correlation functions. Internat. J. Modern Phys. B 4 1003-1037. MR1064758

[16] Kallenberg, O. (1976). Random Measures. Akademie-Verlag, Berlin. MR0431373

[17] KolmogorofF, A. (1977). Grundbegriffe der Wahrscheinlichkeitsrechnung. Springer, Berlin. MR0494348

[18] Lenard, A. (1975). States of classical statistical mechanical systems of infinitely many particles. I. Arch. Ration. Mech. Anal. 59 219-239. MR0391830

[19] Lyons, R. (2003). Determinantal probability measures. Publ. Math. Inst. Hautes Études Sci. 98 167-212. MR2031202

[20] Lytvynov, E. (2002). Fermion and boson random point processes as particle distributions of infinite free Fermi and Bose gases of finite density. Rev. Math. Phys. 14 1073-1098. MR1939761

[21] MacchI, O. (1975). The coincidence approach to stochastic point processes. Adv. in Appl. Probab. 7 83-122. MR0380979

[22] Olshanski, G. (2011). The quasi-invariance property for the Gamma kernel determinantal measure. Adv. Math. 226 2305-2350. MR2739779 
[23] Reed, M. and Simon, B. (1980). Methods of Modern Mathematical Physics. I: Functional Analysis, 2nd ed. Academic Press, New York. MR0751959

[24] Rohlin, V. A. (1949). On the fundamental ideas of measure theory. Mat. Sb. (N.S.) 25 107150. MR0030584

[25] Shirai, T. and Takahashi, Y. (2000). Fermion process and Fredholm determinant. In Proceedings of the Second ISAAC Congress, Vol. 1 (Fukuoka, 1999). Int. Soc. Anal. Appl. Comput. 7 15-23. Kluwer Academic, Dordrecht. MR1940779

[26] Shirai, T. and TAKahashi, Y. (2003). Random point fields associated with certain Fredholm determinants. I. Fermion, Poisson and boson point processes. J. Funct. Anal. 205414 463. MR2018415

[27] Shirai, T. and TaKahashi, Y. (2003). Random point fields associated with certain Fredholm determinants. II. Fermion shifts and their ergodic and Gibbs properties. Ann. Probab. 31 1533-1564. MR1989442

[28] Simon, B. (2011). Trace Ideals and Their Applications, 2nd ed. Mathematical Surveys and Monographs 120. American Mathematical Society, Providence, RI. MR2154153

[29] SinAĬ, YA. G. (1982). Theory of Phase Transitions: Rigorous Results. International Series in Natural Philosophy 108. Pergamon Press, Oxford. Translated from the Russian by J. Fritz, A. Krámli, P. Major and D. Szász. MR0691854

[30] Soshnikov, A. (2000). Determinantal random point fields. Uspekhi Mat. Nauk 55 107-160. MR1799012

[31] Tracy, C. A. and Widom, H. (1994). Level-spacing distributions and the Airy kernel. Comm. Math. Phys. 159 151-174. MR1257246

[32] Tracy, C. A. and Widom, H. (1994). Level spacing distributions and the Bessel kernel. Comm. Math. Phys. 161 289-309. MR1266485

Institut de Mathématiques De MARSEILle 39 RUE F. JOLIOT-CURIE

MARSEILLE 13013

FRANCE

E-MAIL: bufetov@mi.ras.ru

alexander.bufetov@univ-amu.fr 